\title{
Suppression without inhibition: A novel mechanism in the retina accounts for saccadic suppression
}

\author{
Saad Idrees ${ }^{1,2}$, Matthias-Philipp Baumann ${ }^{1}$, Maria M. Korympidou 1,2,3, \\ Timm Schubert ${ }^{1,3}$, Alexandra Kling ${ }^{4}$, Katrin Franke ${ }^{3,5}$, Ziad M. Hafed ${ }^{1}$, \\ Felix Franke ${ }^{6,7,8 *}$, Thomas A. Münch ${ }^{1,3 *}$
}

\author{
${ }^{1}$ Werner Reichardt Centre for Integrative Neuroscience, University of Tübingen, 72076 Tübingen, \\ Germany \\ ${ }^{2}$ International Max Planck Research School, University of Tübingen, 72074 Tübingen, Germany \\ ${ }^{3}$ Institute for Ophthalmic Research, University of Tübingen, 72076 Tübingen, Germany \\ ${ }^{4}$ Department of Neurosurgery, Stanford School of Medicine, Stanford, CA 94305, USA \\ ${ }^{5}$ Bernstein Center for Computational Neuroscience, University of Tübingen, 72076 Tübingen, \\ Germany \\ ${ }^{6}$ Bio Engineering Laboratory, ETH Zürich, 4058 Basel, Switzerland \\ ${ }^{7}$ Institute of Molecular and Clinical Ophthalmology Basel, 4031 Basel, Switzerland \\ ${ }^{8}$ Faculty of Science, University of Basel, 4056 Basel, Switzerland \\ *Correspondence: felfranke@googlemail.com (F.F.), thomas.muench@gmail.com (T.A.M.)
}




\section{Abstract}

Visual perception remains stable across saccadic eye movements, despite the concurrent strongly disruptive visual flow. This stability is partially associated with a reduction in visual sensitivity, known as saccadic suppression, which already starts in the retina with reduced ganglion cell sensitivity. However, the retinal circuit mechanisms giving rise to such suppression remain unknown. Here, we describe these mechanisms using electrophysiology in mouse, pig, and macaque retina, 2-photon calcium imaging, computational modeling, and human psychophysics. We find a novel retinal processing motif underlying retinal saccadic suppression, "dynamic reversal suppression", which is triggered by sequential stimuli containing contrast reversals. This motif does not involve inhibition but relies on nonlinear transformation of the inherently slow responses of cone photoreceptors by downstream retinal pathways. Two further components of suppression are present in ON ganglion cells and originate in the cells' receptive field surround, highlighting a novel disparity between ON and OFF ganglion cells. Our results are relevant for any sequential stimulation encountered frequently in naturalistic scenarios.

Keywords: retina, ganglion cell, cone photoreceptor, saccadic suppression, computational model, sequential stimuli, rapid image shifts, perception, dynamic vision, visual processing 


\section{Introduction}

Vision appears as a continuous and coherent process. This is a striking achievement of the visual system, considering that the visual flow acrsoss the retina is not continuous, but governed by frequent and sudden changes, irregularities, and disruptions. As a consequence of this active vision, i.e. the process of active exploration of the visual environment, the meaningful images falling onto the retina are only brief snapshots of the world, interrupted by blinks and rapid motion. The most prominent cause for such disruptions are eye movements. Saccades, for example, are critical for efficiently sampling the visual world (Schütz et al., 2011; Tatler et al., 2010; Yarbus, 1967), which is particularly true for species in which high visual resolution is limited to a small fraction of the overall visual space, such as the foveal region in primates. On the other hand, as a result of saccades, the number of photons falling onto a given area of the retina can change by several orders of magnitude within tens of milliseconds, causing sudden and frequent visual transients of local intensity across the entire retina. From the perspective of the retina, saccades are therefore equivalent to robust visual stimuli, and they are a powerful model for a very profound question of visual neuroscience: How does the visual system extract robust information from the "meaningful" snapshots of the world, in the face of frequent, strong, and disruptive other input?

Perceptually, saccadic disruptions are minimized by reducing the sensitivity of the visual system to new input around the time of saccades - a phenomenon known as saccadic suppression. While this phenomenon has been extensively characterized over the past few decades (Beeler, 1967; Bremmer et al., 2009; Idrees et al., 2020; Krekelberg, 2010; Matin, 1974; Volkmann, 1986; Wurtz, 2008; Zuber and Stark, 1966), its underlying mechanisms still remain unclear. Several electrophysiological studies have shown neural correlates of saccadic suppression throughout the visual system, namely a modulation of neural activity and/or sensitivity around the time of saccades (Bremmer et al., 2009; Chen and Hafed, 2017; Hafed and Krauzlis, 2010; Ibbotson et al., 2008; Kleiser et al., 2004; Krekelberg, 2010; Wurtz, 2008). These observations have often been interpreted to be caused by active suppressive signals originating from (pre-) motor areas, such as corollary discharge signals related to the saccadic eye movement command (Bremmer et al., 2009; Diamond et al., 2000; Duffy and 
Lombroso, 1968; Ross et al., 2001; Zuber and Stark, 1966). Most studies investigating the mechanisms of saccadic suppression have therefore focused on cortical or subcortical neuronal recordings and/or on behavioral measures of perceptual state, largely neglecting the consequence of visual processing in early visual pathways, for example in the retina.

The retina is an independent signal processing "front end" in the visual system, before visual information is sent along the optic nerve to higher brain areas. Consequently, image processing triggered by visual transients, such as those that naturally occur during active vision, including saccades, could potentially lead to altered retinal output. Retinal signal processing could therefore contribute to perceptual saccadic suppression. Some studies have investigated how the retina processes information in the context of spatio-temporal dynamics that occur during natural visual behavior (Appleby and Manookin, 2019; Baccus et al., 2008; Berry et al., 1999; Chen et al., 2013; Fried et al., 2002; Garvert and Gollisch, 2013; Geffen et al., 2007; Gollisch, 2013; Münch et al., 2009; Tikidji-Hamburyan et al., 2015; Wei, 2018; Zaghloul et al., 2007; Zhang et al., 2012), including saccades (Amthor et al., 2005; Barlow et al., 1977; Enroth-Cugell and Jakiela, 1980; Krishnamoorthy et al., 2017; Krueger and Fischer, 1973; Noda and Adey, 1974; Passaglia et al., 2009; Roska and Werblin, 2003). A retinal neural correlate of perceptual saccadic suppression has recently been shown by a previous study from our labs (Idrees et al., 2020). There, we showed that the retinal output is indeed altered by saccade-like image shifts. In most mouse and pig retinal ganglion cells (RGCs) that we recorded from, responses to brief probe flashes were suppressed when preceded by saccade-like image displacements across the retina. This retinal saccadic suppression had properties consistent with the perceptual suppression of probe flashes reported by human subjects using similar images, and following either real or simulated saccades. In fact, we already observed elementary properties of perceptual saccadic suppression, such as its dependency on background scene statistics, at the level of the output of the retina, providing strong evidence of a retinal mechanism directly contributing to perceptual saccadic suppression.

In this study, we describe such a mechanism. We experimentally mimicked the visual flow resulting from saccades and recorded the neural activity of the output neurons of the retina (RGCs) from ex vivo retinae of mice, pigs, and macaque monkeys. We found 
that retinal saccadic suppression was the result of multiple mechanisms. The major component originated from a novel visual processing motif within an RGC's receptive field center, which we call "dynamic reversal suppression". Dynamic reversal suppression does not depend on any inhibitory signals; it results from a combination of inherent response properties of cone photoreceptors and non-linear processing of cone output by downstream pathways in the retina. Two further components of suppression originated from beyond the RGC's receptive field center, only one of them driven by GABAergic inhibition. Interestingly, these two additional components were observed primarily in ON RGCs, highlighting a novel disparity between ON and OFF type RGCs. Perhaps one of the most intriguing outcomes of this study, also consistent with observations of perception (Idrees et al., 2020), is that the suppressive effects observed in RGCs are not exclusively triggered by saccades, but occur for many scenarios involving sequential visual stimulation, which are ever-present during natural vision. Therefore, while the results described here are crucial for understanding the mechanisms of saccadic suppression, they also elucidate more general mechanisms of retinal signal processing across any time-varying visual input over short time scales (10 $\mathrm{ms}$ to $1 \mathrm{~s}$ ).

\section{Results}

\section{Experimental Approach}

We measured the modulation of retinal ganglion cell (RGC) output following saccade-like changes of the visual input with a variety of different light stimulation strategies (Fig. S1). The basic experimental paradigm was similar to that described in (Idrees et al., 2020). Briefly, we recorded spiking activity of RGCs in isolated ex vivo mouse retinae using both high-density and low-density multi-electrode arrays (MEAs).

Each retina was exposed to a background texture having one of several possible spatial scales that defined its spatial spectrum ("fine" to "coarse", Methods, Fig. S2). We simulated saccade-like image displacements by rapidly translating the texture globally across the retina (Methods; Fig. 1a). Most RGCs responded robustly to such saccade-like texture displacements (see Fig. $1 \mathrm{~b}$ for responses of example $\mathrm{ON}$ and OFF RGCs). At different times relative to the saccade-like texture displacements ("saccades" from now on), we presented a brief probe flash (Fig. 1c). We then 
analyzed how the response (spike rate of the RGC) to this probe flash was influenced by the preceding saccade, by comparing it to the response to the flash presented in isolation (baseline). To quantify RGC response modulation, we calculated a modulation index (Methods) as follows: we first isolated the flash-induced response component by subtracting the saccade-only response (e.g., Fig. 1b) from the response to the composite saccade-flash stimulus (e.g., Fig. 1c). Based on this flash-induced response component (Fig. 1d), we calculated the modulation index as $\left(r_{d}-r_{b}\right) /\left(r_{d}+r_{b}\right)$. Here, $r_{\mathrm{d}}$ is the peak response to the probe flash presented with a delay $d$ relative to saccade onset, and $r_{b}$ is the baseline (peak response to the flash presented $\sim 2 \mathrm{~s}$ after the saccade). This modulation index is negative when flash-induced responses are suppressed (Fig. 1d shows, on the horizontal dashed line, the modulation index for the responses at each flash-time). In yet further recordings we applied various manipulations to this base paradigm to probe for the mechanisms underlying modulation of RGC responses following saccades. To generalize our findings across other species, we also performed similar analyses of pig and macaque RGC data.

\section{Similarities and differences in retinal saccadic suppression across ON and OFF type RGCs}

Suppression was robust across most RGCs that we recorded from, consistent with what we reported previously (Idrees et al., 2020). Here, we more closely inspected functionally different RGCs. Specifically, throughout this study, we divided RGCs into ON and OFF types, i.e. into RGCs responding best to light increments or decrements, respectively (Methods). We always quantified the modulation index defined above for ON RGCs based on their responses to bright probe flashes and for OFF RGCs based on their responses to dark probe flashes (Fig. 1c, d). Flash responses following a saccade were suppressed in both ON and OFF RGCs, as seen in Fig. 1d for example cells. Fig. $1 \mathrm{e}$ shows the temporal profile of the mean population modulation index, and Fig. S3 the underlying population data. Suppression was consistently stronger for coarser background textures (Figs. 1e, S3), for both ON and OFF RGCs. This is consistent with (Idrees et al., 2020), where we showed that this dependency on the texture can be explained by the distinct statistics of luminance and contrast changes when coarse or fine textures move across the RGCs' receptive fields. However, a striking difference existed in suppression recovery times: OFF RGCs recovered by 
$\sim 350$ ms after saccade onset, whereas ON RGCs recovered only by $\sim 1$ s. Similar results were obtained under scotopic conditions for coarse textures, while suppression for fine textures was very weak (Fig. S4; all other mouse retina data was recorded at mesopic conditions). In general, the presence of post-saccadic suppression of probe flash responses in both ON and OFF type RGCs suggests a common mechanistic theme across these cell types (Idrees et al., 2020). On the other hand, the different recovery times indicate either additional suppressive mechanisms in ON RGCs or additional recovery mechanisms in OFF RGCs.

\section{Spatial origin of retinal saccadic suppression}

\section{Global component of suppression}

To probe the mechanisms underlying suppression and its differences across $\mathrm{ON}$ and OFF type RGCs, we first examined the spatial origin of suppression. We hypothesized that suppression of flash responses was caused by circuits detecting rapid global shifts across the retina. Typically, these circuits include a lateral network of interneurons, communicating with RGCs even from beyond their classical center-surround receptive field (i.e., from their periphery, or far surround) (Lin and Masland, 2006; Roska and Werblin, 2003). To test whether suppression was caused by such circuits, we modified the spatial layout of the paradigm: we placed a square mask of $1000 \times 1000 \mu \mathrm{m}^{2}$ (Fig. 2a, right) to restrict the saccades to the periphery of an RGC's receptive field. Similar to the previous experiments, the probe flash was either a dark or bright flash presented over the entire retina, including the masked region. Figure $2 b$ shows the mean population modulation indices of ON RGCs (top) and OFF RGCs (bottom) from these experiments (Fig. S5 depicts the underlying population data and shows responses of representative ON and OFF RGCs from these experiments). In OFF RGCs, responses to full-field probe flashes were no longer suppressed when saccades were restricted to the periphery. The responses of ON RGCs, on the other hand, were still suppressed in this condition. The resulting suppression was however weaker and shorter-lived (recovered by $350 \mathrm{~ms}$ ) than with full-field saccades. These observations (Figs. 2b, S5c) were robust across ON and OFF RGCs whose receptive fields were completely contained within the mask (Fig. S5b). 
We will refer to this component of suppression in ON RGCs, which originates from the periphery, as the "global component" from now on. Such spatially far-reaching inhibition is often mediated through GABAergic wide-field amacrine cells. We tested this hypothesis by blocking $\mathrm{GABA}_{A}$ receptors. Indeed, in the periphery saccade condition, the modulation index for most ON RGCs was around 0 in the presence of the GABAA receptor antagonist SR-95531 (Fig. 2b, S5d). These results suggest that this short-lived global component of suppression is caused by inhibition via GABAergic amacrine cells, perhaps similar to the polyaxonal amacrine cells described previously (Baccus et al., 2008; Ölveczky et al., 2003; Roska and Werblin, 2003). Thus, while suppression is indeed partially caused by circuits detecting global changes across the retina, those circuits seem to act predominantly on ON RGCs, and even there, they only account for a fraction of the total suppression observed with full-field saccades (without mask), which lasts longer. Other, probably more local sources of suppression must exist that account for most of the suppression in ON RGCs and all of the suppression in OFF RGCs.

\section{Local components of suppression}

To understand the more local components of suppression, we used different analyses and manipulations of the main experimental paradigm. As we will see below, the more local components can be subdivided into a "central" and a "surround" component. First, we eliminated the global component, by repeating our normal full-field saccade paradigm in the presence of GABA receptor blockers. The suppression profile of both ON and OFF RGCs was only weakly affected upon blocking GABAA,C receptors $(5 \mu \mathrm{M}$ SR-95531 and $100 \mu \mathrm{M}$ Picrotoxin; Fig. 2c, S6a). Since the GABA-block eliminates the global component of suppression, the remaining more local components did not seem to rely on GABAergic inhibition. Also, this suggests that the local components dominate retinal saccadic suppression under full-field conditions. We then also blocked glycine receptors ( $1 \mu \mathrm{M}$ of Strychnine; Fig. $2 \mathrm{~d}$, S6b) to test if the local components of suppression were caused by local inhibition via glycinergic pathways. Here again, the suppression profiles of both ON and OFF RGCs were only weakly affected upon blocking glycine receptors in combination with blocking $\mathrm{GABA}_{\mathrm{A}, \mathrm{C}}$ receptors. Therefore, inhibitory synaptic interactions are not the major mechanism behind the local components, which dominate suppression of RGCs. 
Next, we tested whether these local components originated from within the receptive field center. For this, we modified the spatial layout of our paradigm to exclude saccades from the very center of the receptive field. Simply reducing the size of our mask would have severely decreased the number of simultaneously recorded cells located inside the mask, and we therefore resorted to a different strategy: saccades and flashes were presented in small square regions spread across the retina, separated by gaps kept at mean luminance (“checkerboard mask", Fig. 2e). In one condition (Fig. 2e, left), we presented saccades and flashes in all regions of the checkerboard mask; in the other condition (Fig. 2e, right), saccades and flashes were presented in alternate regions. With this second arrangement, saccades were excluded from at most $\sim 300 \times 300 \mu \mathrm{m}^{2}$ of a cell's receptive field center, even if that cell was perfectly centered on a non-saccade region. Flashes were presented in the set of regions that included the square region covering the receptive field center of the analyzed RGC (Fig. S7a).

Probe flash responses following saccades were suppressed in both ON and OFF RGCs when the saccade and flash were presented in all regions (Fig. 2f, thick lines; Fig. S7c; see Fig. S7b for example cells), consistent with the suppression observed after full-field saccades (Figs. 1, S3). When saccades were excluded from the receptive field center, and were presented in alternate regions to the flash, the flash responses were no longer suppressed in OFF RGCs (Fig. 2f, bottom, thin line), even though these cells showed spiking responses to saccades themselves (Fig. S7b). In fact, flash responses were even enhanced. This suggests that the local component of suppression in OFF RGCs arises fully from within the receptive field center ("central component"). This highly localized origin of suppression in OFF RGCs was further confirmed by additional analysis of the large mask experiments (see Figs. S7d, e). In ON RGCs, on the other hand, suppression persisted (Fig. 2f, top; Fig. S7c), even though a loss in suppression was apparent for flashes presented immediately after the saccade, at 117 and $150 \mathrm{~ms}$ (marked with an arrow in Fig. 2f). This suggests that in ON RGCs, part of the early suppression originates from the "central component". The leftover suppression during these early time points might be explained by the global component of suppression, described above (Fig. 2b, S5c), which should also be triggered under this experimental setting. However, since the global component also 
recovers quickly (by $350 \mathrm{~ms}$, Fig. 2b, S5c), the persisting suppression at the later time points (350 ms and beyond) needs to originate from yet another source beyond the receptive field center. We call this the "surround component", and it may originate from the ON RGCs' immediate surround, which also experiences the saccade under this experimental setting. Therefore, in ON RGCs, the local component of suppression can be divided into a central and a surround spatial component.

\section{Summary of retinal saccadic suppression spatial origins}

In summary, our data suggests that retinal saccadic suppression is mediated by at least three components with distinct spatial origins and temporal properties (Fig. 3): a central, surround, and global component. Suppression in OFF RGCs is mediated exclusively by the central component, which originates from the cell's receptive field center and is characterized by fast onset and fast recovery (by $350 \mathrm{~ms}$ after saccade onset). In ON RGCs, we most directly observed the global component (Figs. 2b, S5a). It extends into the periphery and its timing is similar to the central component in OFF RGCs. Only this global component is affected by blocking GABA receptors (Figs. 2b, S5b). During full-field saccades, removing this component by blocking GABA receptors has little effect on the overall suppression (Figs. 2c, 2d, S7), suggesting a more dominant role of the remaining components. The central component in ON RGCs can only be observed by the loss in suppression for early flashes when saccades are excluded from the receptive field center (marked with an arrow in Fig. 2f, top). Its full duration and time course are obscured by the concurrently acting global and surround components. However, given the identical pharmacological dependencies and spatial origins, it is plausible that the central component is symmetric across ON and OFF RGCs with a common underlying mechanism. Therefore, the longer suppression in ON RGCs can neither be attributed to the central nor global components. It likely originates from the immediate surround of the receptive field. This surround component is long lasting (recovers by $\sim 1 \mathrm{~s}$ ) and has a slow onset (Figs. 2f, S3).

\section{Suppression is triggered by interaction between consecutive stimuli of opposite polarity}

We previously showed (Idrees et al., 2020) that retinal and perceptual saccadic suppression not only occur after texture displacements, but also after instantaneous 
texture jumps and structure-free uniform luminance steps. These observations suggested that saccadic suppression is the consequence of rather general mechanisms in which the response to a second stimulus (here: probe flash) gets suppressed by a previous visual transient (caused by saccades or luminance steps). In the following, we apply additional analysis to the luminance-step paradigm (Fig. S1b) dataset of (Idrees et al., 2020), to investigate how the polarity and strength of the visual transients affect the suppression of ON and OFF RGCs.

Similar to all previous experiments, we analyzed the modulation index of ON and OFF RGCs separately, using bright probe flashes to analyze ON RGCs and dark probe flashes for OFF RGCs. Consistent with the suppression after texture displacements (Figs. 1e, S3), responses to flashes after luminance steps were strongly suppressed in both ON and OFF RGCs, and ON RGC suppression outlasted suppression in OFF RGCs (Fig. 4a, S8a). The two seemingly different experimental paradigms may therefore trigger similar mechanisms in the retina.

We hypothesized that the response to a luminance step might strongly activate RGCs, so that the response to a subsequent probe flash would drive the cells into adaptation or saturation, effectively resulting in suppressed flash responses. At least for the local components of suppression, this could be a viable mechanism as suppression is not caused by inhibitory synaptic interactions. If this was indeed the case, then positive-contrast luminance steps would suppress responses to bright flashes in ON RGCs, and negative-contrast luminance steps would suppress responses to dark flashes in OFF RGCs. To test this, we separately analyzed the effects of positive- and negative-contrast luminance steps on probe flash responses (Fig. 4b). Surprisingly, the resulting effects were contrary to our adaptation/saturation hypothesis: the responses of ON RGCs to bright probe flashes were only weakly suppressed after positive-contrast luminance steps (Fig. 4b, left), but strongly suppressed following negative-contrast luminance steps (Fig. 4b, right). Similarly, responses of OFF RGCs to dark probe flashes were weakly suppressed by negative-contrast luminance steps (Fig. 4b, right), but strongly suppressed by positive-contrast luminance steps (Fig. 4b, left). Fig. S8 shows the underlying population data for these experiments, and Figs. S9a, b show the spiking response of a representative ON and OFF RGC, respectively. While ON RGCs did show a small component of suppression in support 
of our adaptation/saturation hypothesis (Fig. 4b left panel, see Fig. S9a left column and Fig. S10a for a detailed analysis), the dominant suppressive effect in both ON and OFF RGCs was caused by luminance steps with the opposite contrast as the subsequent flash.

Such crossover style of suppression would be consistent with mechanisms involving crossover inhibition via amacrine cells (Werblin, 2010), where activation of OFF pathways (here: by the negative-contrast luminance step) would inhibit responses in the ON pathway (here: to the bright probe flash) and vice versa. However, consistent with our earlier experiments (Fig. 2c, d), suppression in ON RGCs still persisted upon blocking $\mathrm{GABA}_{A, C}$ and glycine receptors $(5 \mu \mathrm{M}$ SR-95531, $100 \mu \mathrm{M}$ Picrotoxin and $1 \mu \mathrm{M}$ Strychnine) (Fig. 4c). We could not calculate a modulation index for OFF RGCs under these conditions because they did not respond to brief probe flashes in the presence of the pharmacological agents, and therefore the modulation index was mathematically undefined. However, in our texture displacement experiments, the same pharmacological agents (Fig. 2d) had no substantial effect on OFF RGC suppression. The crossover-style suppression observed in Fig. 4b was therefore unlikely to be caused by classical crossover inhibition pathways involving amacrine cells and $G_{A B A}, C$ or glycine receptors.

\section{Central component of suppression results from cone response kinetics and nonlinearities in downstream retinal pathways}

So far, our experiments suggest that suppression in OFF RGCs (1) is mediated solely by the central component of suppression that originates in the receptive field center (Figs. 2e, S7b-e), (2) is predominantly triggered by the interaction between consecutive stimuli with opposite polarity (Figs. 4b, S8b), and (3) is not caused by inhibitory amacrine cells (Figs. 2c, 2d, S6). Similar conclusions can be drawn for the central component of suppression in ON RGCs. Together, these results restrict the possible cellular substrates for this central suppressive mechanism to the feed-forward pathway in the retina, namely photoreceptors - bipolar cells - RGCs. We wondered whether opposite-polarity stimulus-stimulus interactions could already modulate the responses of photoreceptors themselves. For this, we recorded the output of cones with an intensity-based glutamate-sensitive fluorescent reporter (iGluSnFR) (Marvin 
et al., 2013; Szatko et al., 2020), predominantly expressed in horizontal cells postsynaptic to cone terminals (Methods). We presented a shortened version of the luminance step paradigm in which a homogeneous background alternated between a brighter and darker gray value (Fig. S1C) to induce positive-contrast (+0.4 Michelson contrast) and negative-contrast (-0.4 Michelson contrast) luminance steps. Dark or bright probe flashes (100 ms duration, -0.33 or +0.33 Michelson contrast, respectively) followed the luminance steps at different delays (50, 250, and $2000 \mathrm{~ms}$ ), with the flash at 2000 ms serving as baseline.

The luminance steps caused sustained changes in the cones' glutamate output (Fig. 5a). The transient responses to the probe flashes were superimposed on these glutamate modulations (Fig. 5b). This superposition was mostly linear and did not indicate nonlinear effects such as adaptation or saturation. Therefore, when we isolated the flash responses by subtracting the step responses, the resulting peak flash responses were only weakly affected by the preceding luminance step (Fig. 5c). Thus, at the level of the cone output (Fig. 5b), there was hardly any suppression when only considering the peak of the probe flash responses (Fig. 5c). How does the suppression observed at the level of RGC output arise from effectively linear cone responses? The answer lies in other properties of the cone response, such as its kinetics, which will be captured by downstream retinal pathways.

To demonstrate this, we used a previously published phenomenological model of retinal processing. In the model (Drinnenberg et al., 2018), RGC responses are described by passing a "light stimulus" to model photoreceptors, feeding their output through a set of linear-nonlinear filters to describe the processing in different bipolar pathways (Methods), and eventually converting these filter outputs into RGC spiking. Here, as the first step of analysis, we fitted the parameters of the model cone to reflect our measured data of cone output. The model faithfully explained the observed cone responses (Fig. S11) and gave us the opportunity to calculate cone responses to flashes presented at additional time points. This modeled cone output to step-flash combinations was fed into the model bipolar cells, finally yielding model RGC responses (Fig. 6). In the model, different RGC types can be described by varying the bipolar cell filter properties. We first investigated transient RGC responses (Fig 6a-c) and calculated a modulation index (Fig. 6d) comparable with the modulation index of 
our real RGC data. As a control, we also passed the raw cone output data, instead of the fitted cone model, to the model bipolar cells and found qualitatively the same results (Fig. S12).

The model's ON and OFF RGCs (Fig. 6d) showed crossover-style suppression that was consistent with the suppression of real RGCs under similar luminance step experiments (Fig. 4b): the model's ON RGC showed strong suppression to the bright flashes presented immediately after the negative-contrast luminance step (Fig. 6d, right), while flashes after the positive-contrast luminance step were only weakly affected (Fig. 6d, left). Suppression recovered by $200 \mathrm{~ms}$, consistent with the recovery time for the central component of suppression in real RGCs. Similarly, the model's OFF RGC showed strong suppression of its response to dark flashes presented immediately after the positive-contrast luminance step (Fig. 6d, left); suppression was absent in OFF RGC when the dark flash was presented later or after a negative-contrast luminance step (Fig. 6d). The markers on the curves in Fig. 6d correspond to the time points when the flashes were presented to the cones in the experiments depicted in Fig. 5. Model RGC responses to step-flash combinations and flash-induced responses at these time points are shown in Fig. 6a-c.

What mechanism within the model led to the emergence of the suppressive effect in RGC responses, despite the mostly linear response superposition at the cone output? In the model, the bipolar cells have transient filter properties and are driven predominantly by the instantaneous rate of change in the cone output, i.e. its derivative, rather than by the absolute cone output. The response to a probe flash presented immediately after an opposite-contrast luminance step (50 ms) occurred during the initial phase (ramp) of the cone response to the luminance step (Fig. 5b, columns 2 and 3). This causes a much smaller rate of change in cone output and therefore drives the downstream bipolar cells only weakly, resulting in weak or even completely suppressed model RGC responses (Fig. 6c, columns 2 and 3). On the other hand, flashes presented during the steady state phase of the luminance step response (250 ms and $2000 \mathrm{~ms}$ in Fig. 5b, columns 2 and 3), or flashes presented immediately after (50 ms) a same-contrast luminance step (Fig. 5b, columns 1 and 4) caused larger instantaneous changes in the cone output, and therefore resulted in 
417 relatively stronger spiking (250 ms and $2000 \mathrm{~ms}$ in Fig. 6c; 50 ms in Fig. 6c, columns 4181 and 4$)$.

419 If suppression observed at RGC output did indeed result from the weaker rate of change in the cone output, less transient RGCs should show weaker suppression because they reflect the absolute cone output signal more faithfully. To test this, we varied the linear-nonlinear filter of the model bipolar cell to make the retinal pathway less transient (Methods). Indeed, the resulting crossover-style suppression was weaker in model RGCs with less transient response properties, indicated by the negative modulation index slope in Fig. 6e; seen in the ON RGC suppression after negative-contrast steps (blue lines in row 2) and OFF RGC suppression after positive-contrast steps (red lines in row 1); columns represent different time points sampled. Curious if this effect was also present in our real RGC data, we re-analyzed the data of Fig. 4 and evaluated suppression as a function of RGC transiency (Methods). The result was congruent with the model prediction: suppression was weaker in less transient (more sustained) cells (Fig. 6f).

The analyses above suggest that the central component of retinal saccadic suppression does not have a single site of origin. Instead, it appears to emerge from the relatively slow kinetics of cone responses (such that flash responses ride on the initial rising/falling phase of the cone's step response), combined with temporal properties and nonlinearities of downstream retinal pathways. The downstream pathways shape the exact strength and time course of RGC suppression. We term this novel retinal processing motif "dynamic reversal suppression": "dynamic" because of the necessarily tight temporal link between responses to the two consecutive stimuli; and "reversal" because the response to an event gets suppressed when it reverses the cone output triggered by the first stimulus.

\section{Generalization to other species}

443 Retinal saccadic suppression, at least its central component, was triggered by stimulus-stimulus interactions (Figs. 4, 6, S8), governed by general retinal signal processing, without the need for any specialized "saccadic suppression" circuit. It is likely that such general processing is conserved across species. Indeed, we observed 
quantitatively similar retinal saccadic suppression in pig ON and OFF RGCs (Fig. S13), including the dependency on background texture statistics. Interestingly, like in mouse, OFF RGCs in pig retina also recovered faster than ON RGCs, suggesting that the surround component of suppression was also present in pig ON RGCs. In an additional experiment, we also recorded the activity of RGCs from an ex vivo macaque retina while subjecting it to a shorter version of the luminance step paradigm (Fig. S1b) with fewer conditions than in the original paradigm. Our results (Fig. 7) indicate that macaque RGC responses to probe flashes, following luminance steps, are suppressed in a way similar to mouse retina. However, more data will be required to determine the population trend and for characterizing the dictionary of response modulations in macaque retina.

\section{Downstream visual areas may modulate retinal saccadic suppression}

Given the similarities we previously described between retinal and perceptual saccadic suppression (Idrees et al., 2020), it was tempting to test whether the crossover style of suppression, observed in the retina (Fig. 4b), was also reflected in perception. We therefore conducted human psychophysics experiments where we asked human subjects $(N=5)$ to maintain saccade-free fixation, while we simply changed the luminance of the homogenous background to a brighter (0.3 to 0.56 Michelson contrast) or darker (-0.3 to -0.56 Michelson contrast) background (Fig. 8a; Methods). At random times relative to the luminance step, we presented a dark ( -0.033 Michelson contrast) or bright probe flash (+0.033 Michelson contrast), at one of four locations in the subjects' field of view. At trial end, the subjects were asked to localize the probe flash.

Irrespective of the step $\rightarrow$ flash combination, subjects were strongly impaired in their ability to localize the probe flashes presented around the time of the luminance step (Fig. 8b). Most interesting in this context was the combination of negative-contrast luminance steps with dark probe flashes. In mouse retina, even though few OFF RGCs did show weak suppression to this combination (Fig. S8b, inverted histograms in row 2 ), this effect was virtually absent at the population level (Fig. 4b, right panel). In human perception, however, this combination led to strong suppression (Fig. 8b, right panel). We cannot exclude that stronger retinal suppression to this specific 
combination might be present under different light or stimulus conditions. Another possibility is that it might be more pronounced in the retina of humans and other primate species (Fig. 7). Nonetheless, visual mechanisms of suppression certainly exist in higher visual brain areas (Idrees et al., 2020). Perceptual suppression after same-contrast stimulus combinations may arise from processing in these higher visual brain areas, which may modulate and complement retinal saccadic suppression to achieve robust effects at the perceptual level.

\section{Discussion}

For most RGCs that we recorded, responses to brief probe flashes were strongly suppressed when presented after saccade-like texture displacements across the retina. In fact, similar suppression occurred when texture displacements were replaced by sudden uniform changes in background luminance, suggesting that suppression was caused by rather generic mechanisms, triggered by visual transients across the retina, rather than specialized suppression circuits that react to image motion. We found that the suppression strength depended on four main factors: (1) strength of the visual transients, governed by the statistics of the background texture or the contrast of the luminance step; (2) elapsed time following the visual transient; (3) RGC polarity (ON vs. OFF RGCs); and (4) RGC response properties (RGC transiency). Stronger visual changes, elicited either by coarser textures or larger luminance step contrasts, caused stronger suppression, peaking approximately $50 \mathrm{~ms}$ after the stimulus offset (Figs. 1e, 4). The recovery times depended on RGC polarity: OFF RGCs recovered by 250 - $350 \mathrm{~ms}$ whereas suppression in ON RGCs lasted for up to $1 \mathrm{~s}$ (Figs. 1e, 4). The suppression was stronger in more transient RGCs (Fig. 6e, f). We identified at least three components of retinal saccadic suppression, with distinct spatial origins, defined as central, surround, and global components (Fig. 3). These components were mediated by different underlying mechanisms.

The central component was the only source of suppression in OFF RGCs, and the dominant source in ON RGCs for time points immediately after a full-field saccade or luminance step. This component was short-lived ( 250-350 ms) and originated from a cell's receptive field center. It was triggered by opposite-polarity stimulus-stimulus interactions, which naturally occur during saccades and other forms of sequential 
visual stimulation. The relatively slow temporal dynamics of the cone photoreceptor responses to two opposite-polarity consecutive stimuli were the basis for subsequent non-linear processing, ultimately suppressing RGC response to the second stimulus. Therefore, the central component of suppression cannot be pinpointed to one specific site but seems to be an emergent property. We call this novel processing motif "dynamic reversal suppression". Such a mechanism, where the cone response itself remains linear, but nonetheless forms the basis for subsequent nonlinear response modulation, is clearly different from adaptation (Clark et al., 2013) or desensitization (Baylor and Hodgkin, 1974) mechanisms within the cones, which would evoke nonlinear responses of the cones themselves.

The suppressive mechanism of the central component also suggests that perceptual saccadic suppression is derived, at least in part, from the inherent response properties of photoreceptors, the very first cell in the visual processing cascade. In fact, this early implementation could also explain why we observed suppression in most RGCs we recorded from (Figs. S3, S8, S13), covering a wide spectrum of response properties and therefore presumably many RGC cell types (see Supplementary Fig. 6 of Idrees et al., 2020). Still, our results suggest that the suppression initiated at the level of cone photoreceptors is translated differently by the different parallel pathways in the retina, leading to variability in response suppression at the ganglion cell level (such as the stronger suppression in more transient RGCs, Fig. 6e, f). Further cell type classification will be required to relate the degree of modulation with pathway specificity. The type and degree of modulation might also differ across species, even though we see qualitatively similar suppression in mouse, pig, and macaque RGCs.

ON RGC sensitivity, in addition to this central component, is suppressed by two more components. First, the global component is a fast but short-lived ( $250-350 \mathrm{~ms})$ component, caused by inhibition via GABAergic wide field amacrine cells, triggered by global image changes and carried to the RGC from as far as the cell's periphery (Figs. 2b, S5). This likely belongs to the same class of circuits that suppresses RGC responses to global motion (Baccus et al., 2008; Roska and Werblin, 2003). These circuits were previously suggested to suppress motion awareness during saccades, a phenomenon known as saccadic omission. As indicated by our results, such circuits also contribute towards suppressing RGC sensitivity even after the motion is 
completed (i.e. saccadic suppression). However, since their influence is masked by more local components of suppression during full-field saccades (Fig. 2c, 2d, S6), they are unlikely to account for perceptual saccadic suppression. The global suppressive component mediated by GABAergic inhibition is nevertheless a mechanism to process global visual changes, in addition to the several others (Wei, 2018). It may, for example, play a role in perceptual modulations during smooth pursuit eye movements (Schütz et al., 2011). Here, the central component of suppression will not be triggered in RGCs whose receptive field centers are locked to the tracked object; but these RGCs will still be suppressed by the global component.

The second additional component is the surround component. It seems to act with a delay of $\sim 200 \mathrm{~ms}$ and can last for up to $1000 \mathrm{~ms}$. The spatial origins of this component are not very well understood, but our data indicate that it presumably arises from the immediate surround of a cell's receptive field. Additionally, similar to the central component, it does not rely on $\mathrm{GABA}_{\mathrm{A}, \mathrm{C}}$ or glycine receptors (Fig. 2c, 2d, S6), but the exact mechanisms remain to be explored. Possible mechanisms could involve negative feedback of horizontal cells onto the cones (Drinnenberg et al., 2018; Kemmler et al., 2014). This slower component most likely contributes to the longer recovery times observed in ON cells. Interestingly, visual masking in cat LGN also lasts longer in ON versus OFF cells (Schiller, 1968), which may be a consequence of the effects we describe here in the retina. While this surround component plays an important role in shaping RGC and downstream neural responses following a visual transient, its contribution to perceptual saccadic suppression can also be disputed. This is because, during real saccades, eye-movement related signals (e.g., corollary discharge) shorten the duration of suppression caused by visual mechanisms (Idrees et al., 2020), such that the long-lasting surround component may not critically shape perception. Yet another additional component of suppression, based on saturation-like mechanisms (Fig. 4b left, S10a), was found only in ON RGCs. It is possible that this component originates at the level of bipolar cells, especially because response saturation has been observed predominantly in ON bipolar cells but not in OFF bipolar cells (Schreyer and Gollisch, 2020). In summary, while Fig. 3 summarizes the three spatial components of suppression and their temporal properties, these components in turn can have further sub-components. 
Irrespective of which components of retinal saccadic suppression contribute towards perceptual saccadic suppression, our results show that retinal responses to stimuli following visual transients are modulated concurrently by several mechanisms (Fig. 3). Additional mechanisms might exist under different stimulus conditions. From the perspective of retinal visual feature processing, this would be consistent with how multiple mechanisms concurrently process other visual features in the retina, such as motion (Wei, 2018). The retinal suppression that we studied likely contributes to several other visual phenomena, such as visual masking (Breitmeyer, 2007) or neural adaptation with successive stimuli (Mayo and Sommer, 2008). The similarities between the observations there and the suppression that we observed in the retina suggest that the retina may be a common neural substrate for these seemingly different types of perceptual phenomena, unifying their underlying mechanisms. The retinal processing described here may also contribute towards specialized visualmotor tasks. For example, while threading a needle, microsaccades will jitter the needle and its background across the retina. RGCs exposed to the background will experience stronger visual transients than RGCs exposed to the needle. As a result, RGCs 'seeing' the background might show suppressed responses following microsaccades, perceptually blurring out the background. This relative enhancement of the fixated object by background suppression could be valuable for visual tasks such as object recognition.

It is remarkable that an elementary property of retinal suppression, i.e. its dependence on the scene statistics (Fig. 1e), is clearly preserved all the way to perception (Idrees et al., 2020). However, retinal suppression alone cannot explain all perceptual effects of saccadic suppression. For example, suppression before a saccade (or a luminance step) is observed perceptually (even in the absence of eye movements, Fig. 8), but not in the retinal output (Idrees et al., 2020). Similarly, not all properties of retinal suppression were preserved in perception. The crossover style of suppression observed in the retinal output of mice (Fig. 4b) was not observed perceptually in humans (Fig. 8). The more far-reaching perceptual suppression may be the result of additional processing beyond the retina (Shapley and Enroth-Cugell, 1984). Alternatively, our results may not capture the full array of retinal processing. For one, our stimulus conditions in mouse retina experiments may not have been 
comprehensive enough to capture all retinal suppressive effects. Further, the retinal output of humans and other primate species (Fig. 7) might differ from mouse retina in this respect.

Retinal suppression is only one way that the retina alters its output during dynamic vision. Other forms certainly co-exist, such as brief changes in RGC polarity following peripheral shifts (Geffen et al., 2007) or sensitization of some RGC types following a change in background luminance (Appleby and Manookin, 2019). These and several other studies (Amthor et al., 2005; Barlow et al., 1977; Enroth-Cugell and Jakiela, 1980; Krishnamoorthy et al., 2017; Noda and Adey, 1974; Passaglia et al., 2009; Roska and Werblin, 2003), together with ours, demonstrate the complex image processing capabilities in the retina to facilitate downstream visual processing for the ultimate service of perception during natural vision. Looking forward, the detailed characterization of retinal output provided here paves the way to investigate the visual features that the retina encodes during dynamic vision. Moreover, it also paves the way to investigate the interactions between retinal and extra-retinal (visual and non-visual) mechanisms of saccadic suppression, to further our understanding of how the visual system maintains stability in the face of constant disruptions.

\section{Acknowledgements}

We thank Elisabeth Gustafsson for providing technical support; Andreas Hierlemann for providing the HiDens CMOS MEA system and helping establish our high-density MEA recordings; Roland Diggelmann for helping in setting up the pipeline (including providing code) for automatic spike sorting of high-density MEA recordings; Martin Schenk at the Department of Experimental Surgery, Tübingen, for providing us domestic pig eyes. Our work was supported by funds of the Deutsche Forschungsgemeinschaft (DFG) to the Werner Reichardt Center for Integrative Neuroscience (EXC 307) and to TAM (MU3792/1-1 and MU3792/3-1). TAM received support from the Tistou and Charlotte Kerstan Foundation. SI, TAM and ZMH were also supported by an intra-mural funding program (Project 2013-05) of the Werner Reichardt Center for Integrative Neuroscience. FF was supported by a Swiss National Science Foundation Ambizione grant (PZ00P3_167989) and Swiss National Science Foundation Eccellenza grant (PCEFP3_187001). MPB and ZMH were further funded 
by the SFB 1233 on "Robust Vision" (DFG, project number 276693517). KF was and the Max Planck Gesellschaft (MPG, M.FE.A.KYBE0004).

\section{Author contributions}

640 TAM conceptualized the study; SI and TAM designed the overall study; FF developed 641 and supervised computational modeling; SI, MPB, AK, KF, ZMH, FF and TAM 642 designed experiments; SI performed ex vivo mouse and pig retina experiments; AK performed ex vivo macaque retina experiment; MPB performed human psychophysics experiments; KF performed viral injections; MK performed cone imaging experiments; SI and FF implemented computational model; All authors analyzed and interpreted the data and wrote the manuscript.

\section{Declaration of interests}

648 The authors declare no competing interests.

\section{Data availability}

650 All data presented in this paper are stored and archived on secure institute computers 651 and are available upon reasonable request.

\section{Code availability}

653 All code used for analysis and computational modeling is available upon reasonable 654 request. 
bioRxiv preprint doi: https://doi. org/10.1101/2020.08 21.261198; this version posted September 10, 2020. The copyright holder for this preprint (which was not certified by peer review) is the author/funder, who has granted bioRxiv a license to display the preprint in perpetuity. It is made available under aCC-BY-NC-ND 4.0 International license.

\section{Figure 1}

a Saccade paradigm

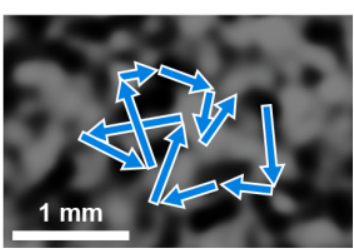

b Response to saccades-alone

ON RGC
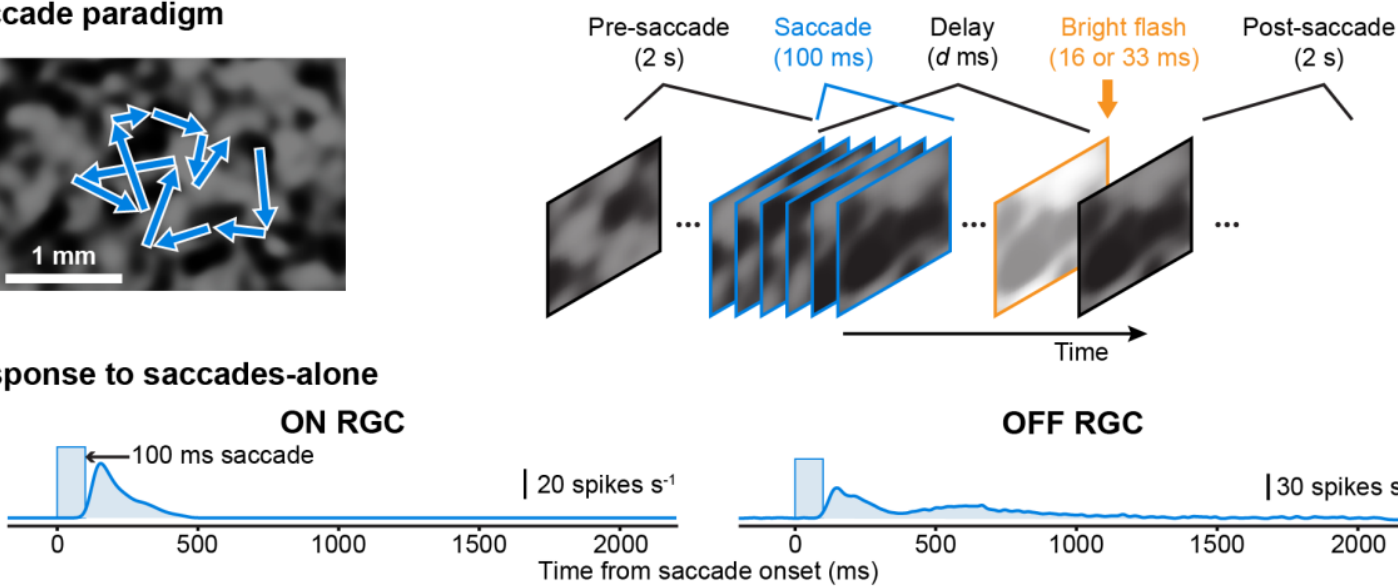

\section{Response to saccades followed by probe flashes}
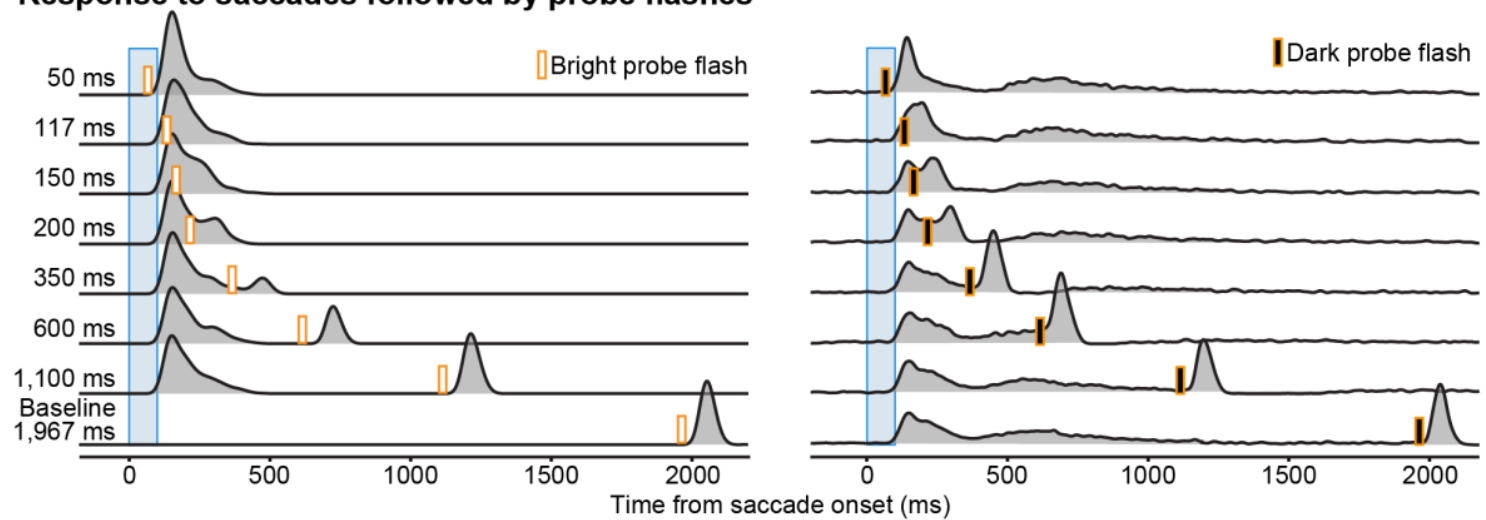

d Flash-induced responses

Modulation 낭유요 N indices
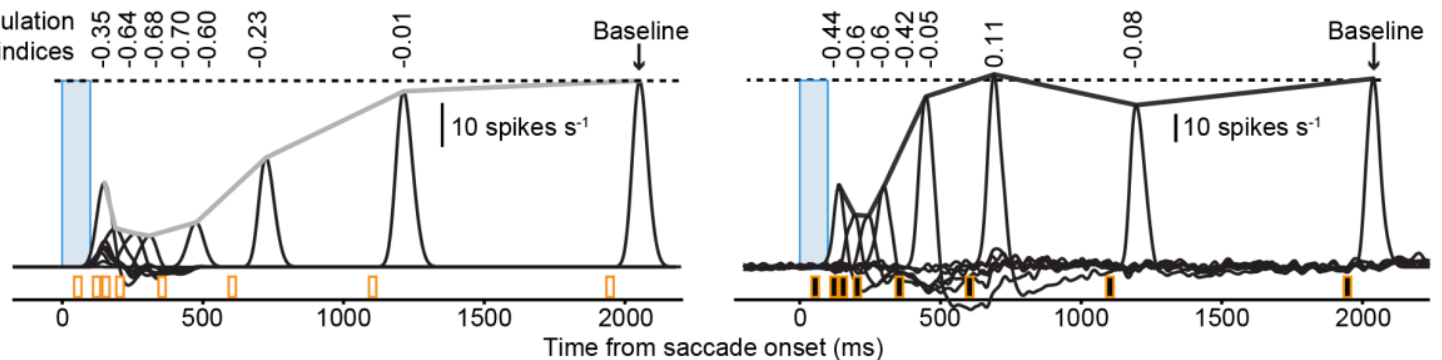

e Modulation index (population) 
658 Figure 1 Similarities and differences in retinal saccadic suppression across ON and 659 OFF RGCs.

660 a. RGC action potentials were recorded from ex vivo retinae placed on multielectrode 661 arrays. Saccades were mimicked by displacing a texture projected onto the retina 662 (blue arrows in left panel indicate texture displacement paths). The texture remained 663 static for $2 \mathrm{~s}$ and was then displaced over $100 \mathrm{~ms}$ (blue outlines) followed by a brief 664 probe flash (here bright probe flash is depicted, orange outline). Each trial consisted 665 of 39 such successive saccade-flash sequences (Fig. S1a).

666 b,c. Average activity (firing rate) of an example ON RGC (left column) and OFF RGC 667 (right column) to 39 saccade sequences not followed by a probe flash (b), and to 39 668 saccade sequences followed by probe flashes at different delays after saccade onset 669 (c). Blue window: timing of saccades; orange markers: timing of probe flashes.

670 d. Isolated flash-induced responses (firing rate) of the same RGCs obtained by 671 subtracting responses to saccades-alone (b) from responses to saccades followed by 672 probe flashes (c). Lines connecting the response peaks highlight the time courses of 673 retinal saccadic suppression relative to baseline flash-induced responses. Numbers 674 above each response peak represent the modulation index which quantifies how much 675 the probe flash response is modulated by the preceding saccade (Methods, negative 676 modulation indices correspond to suppressed flash-induced responses).

677 e. Population modulation index (mean \pm s.e.m.) of ON (light gray) and OFF (dark gray) 678 RGCs, for different background textures with different spatial scales (left to right: fine 679 to coarse). The number of ON and OFF RGCs in the population varied between 68 680 and 574 for different flash times and textures (see Fig. S3 for exact numbers and 681 relevant statistics). Hash symbols: significant modulation difference between ON and OFF RGCs ( $p<10^{-4}$, two-tailed Wilcoxon rank-sum test). 


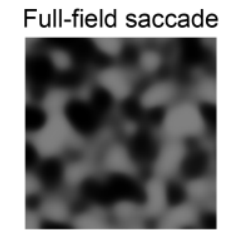

b

ON RGCs

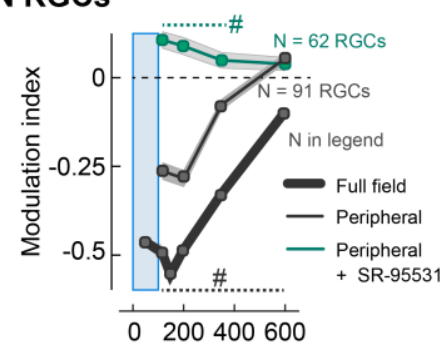

OFF RGCs

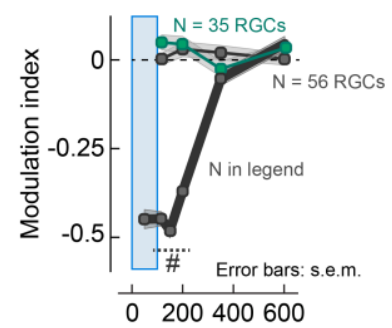

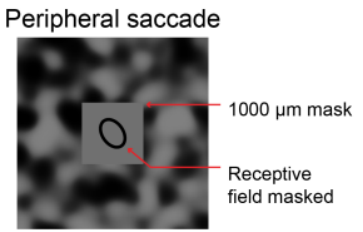
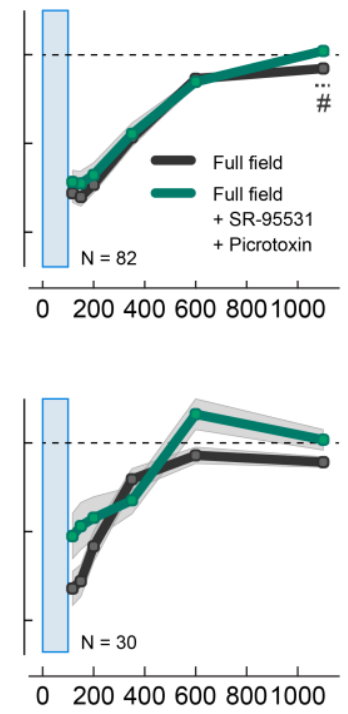

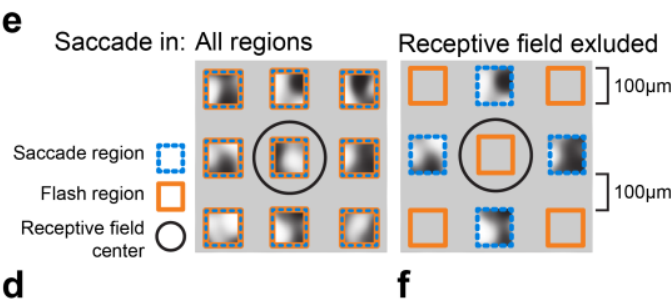

d
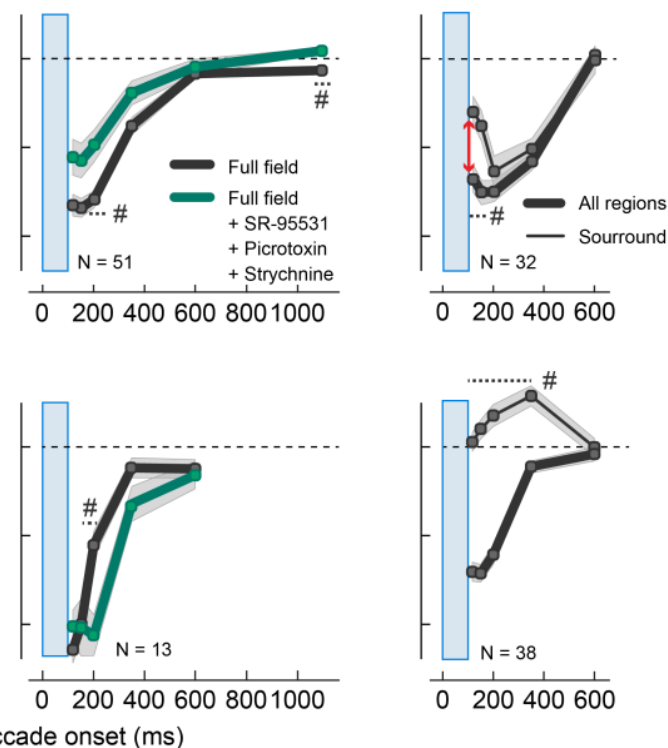

Figure 2 Spatial origins of retinal saccadic suppression.

a. Spatial layout of the visual stimulation paradigm used in experiments to probe the global component of suppression. Saccades were presented either full-field (left; same as in Fig. 1) or in the periphery (right), where a $1000 \times 1000 \mu \mathrm{m}^{2}$ mask (intensity: mean luminance of texture) covered at least 2- $\sigma$ of the 2D Gaussian fit to the RGC receptive fields (Fig. S5b).

b. Population modulation index (mean \pm s.e.m.) of ON (top) and OFF (bottom) RGCs for full-field saccades condition (thick gray lines, same as Fig. 1e rightmost panel; $\mathrm{N}=68$ to 574 RGCs (see Fig. S3 for exact numbers)); periphery saccades condition (thin gray lines; $\mathrm{N}=91 \mathrm{ON}$ RGCs, $\mathrm{N}=56$ OFF RGCs); and periphery saccades condition in the presence of $\mathrm{GABA}_{A}$ receptor blocker $(5 \mu \mathrm{M}$ SR-95531; green lines; $\mathrm{N}=62$ ON RGCs, $\mathrm{N}=35$ OFF RGCs). Blue window shows the timing of the saccade. In these experiments, we used a coarse background texture (300 $\mu \mathrm{m}$ spatial scale). Timing of probe flashes: 50 and 150 (only for full-field saccade), 117, 200, 350, 600 and $2100 \mathrm{~ms}$ (baseline) after saccade onset. for full-field saccades without any pharmacological agents (gray lines; $N=82$ ON RGCs, $N=30$ OFF RGCs) and with GABAA,C receptor blockers $5 \mu \mathrm{M}$ SR-95531 + $100 \mu \mathrm{M}$ Picrotoxin (c; green lines), and for a subset of RGCs where we additionally 
blocked glycine receptors using $1 \mu \mathrm{M}$ Strychnine (d; green lines; $\mathrm{N}=51$ ON RGCs, $\mathrm{N}=13$ OFF RGCs). In these experiments, we used a coarse background texture (150 $\mu \mathrm{m}$ spatial scale). Probe flashes were presented at $117 \mathrm{~ms}, 150,200,350,600,1100$ and 2100 (baseline) after saccade onset.

e. Spatial layout of the visual stimulation paradigm used in experiments to probe local components of suppression. Saccades and flashes were presented in $100 \times 100 \mu \mathrm{m}^{2}$ square regions, separated by $100 \mu \mathrm{m}$ gaps with mean overall luminance. Left: Saccades and flashes were presented in all regions. Right: Saccades and flashes were presented in alternate regions; only cells with receptive fields (RFs) in the non-saccade regions (orange) were analyzed (black ellipse: 1- $\sigma$ of the 2D Gaussian fit to an example RGC receptive field). Consequently, saccades were excluded from at most $\sim 300 \times 300 \mu \mathrm{m}^{2}$ of a cell's RF center. In these experiments, we used a coarse background texture (150 $\mu \mathrm{m}$ spatial scale).

f. Population modulation index (mean \pm s.e.m.) of ON (top; $N=32$ ) and OFF (bottom;

$719 \mathrm{~N}=38$ ) RGCs for saccades and flashes in all regions (thick lines) or saccades excluded from RGC RF center (thin lines). Red arrow indicates significant loss in suppression in ON RGCs for early flashes at 117 and $150 \mathrm{~ms}$ upon excluding saccades from RF center ( $p=0.0016$ and $p=0.002$ respectively; two-tailed Wilcoxon rank-sum test). ( $p<0.01$, two-tailed Wilcoxon rank-sum test). 
ON RGC

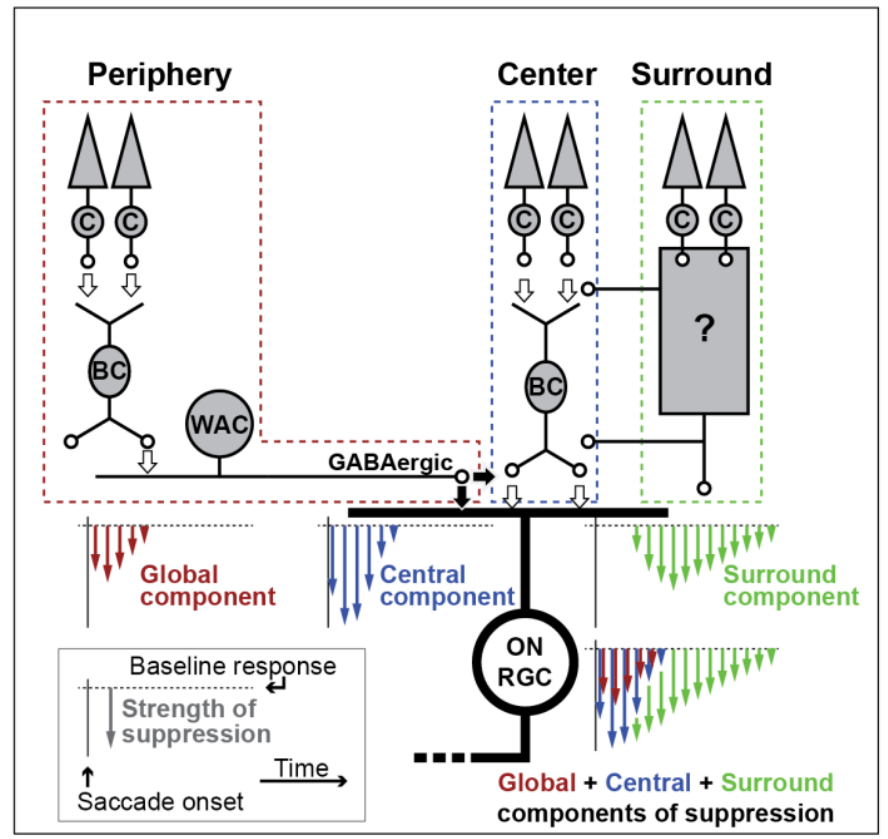

OFF RGC

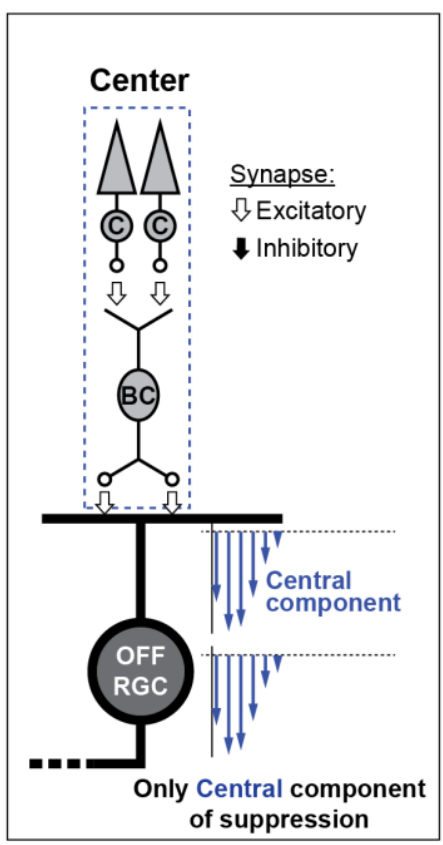

Figure 3 Schematic summarizing the spatial origins of retinal saccadic suppression.

Saccadic suppression in OFF RGCs (right) is mediated primarily by the central component of suppression (blue) that originates from the cells' receptive field center. ON RGCs (left) get suppressed from two additional components: First, the fast but short-lived global component (red), mediated by GABAergic inhibition, that originates from as far as the cells' periphery. This global component has a similar temporal profile as the central component. It is however weaker than the central component and acts in parallel to it, indicated by the red arrows parallel to blue arrows in the total suppression schematic. Second, the delayed but long-lasting surround component (green), which might originate from the cell's immediate surround. The central component and surround component do not depend on classical GABAergic or glycinergic inhibitory pathways. The differences in the suppression recovery time in ON and OFF RGCs was mainly due to this surround component acting on ON RGCs. Inset shows the legend for arrow schematics. Length of the arrows represent suppression strength; spread of the arrows show the temporal profile of suppression. 
Figure 4

a

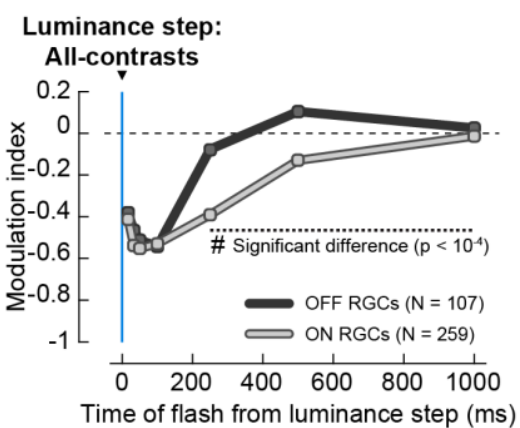

b

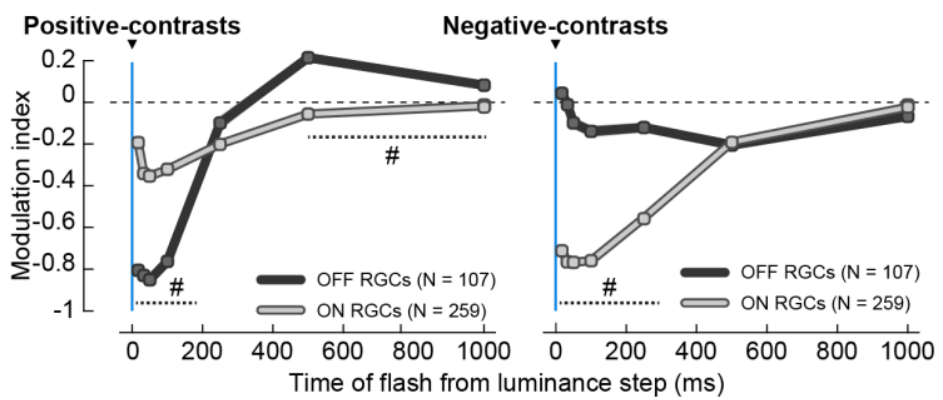

C

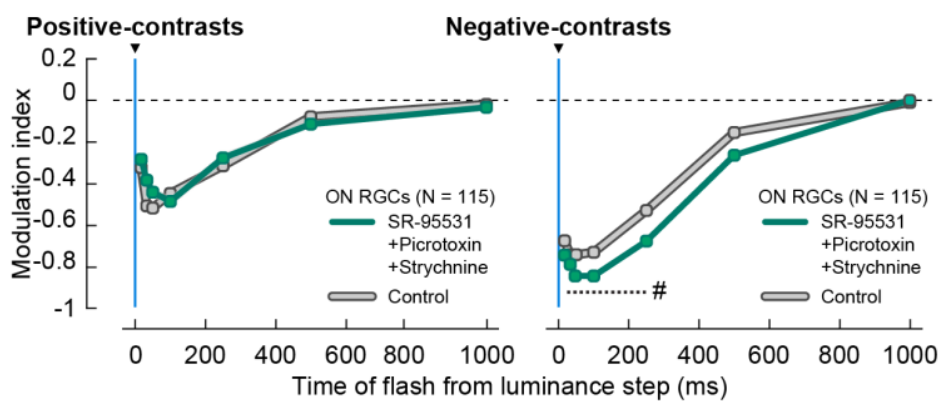

\section{Figure 4 Suppression following luminance steps.}

a. Population modulation index (mean \pm s.e.m.) of ON (light gray, $N=259$ ) and OFF (dark gray, $N=107$ ) RGCs for probe flashes following luminance steps (blue line). Modulation index for each RGC was based on its average response to 56 or 156 luminance step sequences (Fig. S1b) spanning a contrast range of -0.5 to +0.5 Michelson contrast (Methods). Probe flashes were presented at $17 \mathrm{~ms}, 33,50,100$, 250, 500, 1000 and 2000 (baseline) after luminance steps. Probe flash responses were suppressed in both ON and OFF RGCs, with similar time course and recovery as in the saccade paradigm with textures (Fig. 1e). Error bars are not visible due to small s.e.m.

b. Same as in a, except that the modulation index for each RGC was separately based on average responses to probe flashes after positive-contrast luminance steps (left panel; +0.03 to +0.5 Michelson contrast), and after negative-contrast luminance steps (right panel; -0.03 to -0.5 Michelson contrast). Underlying population data is shown in 762 Fig. S8.

c. Same as in $\mathbf{b}$, for a subset of ON RGCs $(N=115)$ in control conditions (light gray lines) and with $\mathrm{GABA}_{A, C}$ and glycine receptors blocked (green lines; cocktail of $5 \mu \mathrm{M}$ SR-95531, $100 \mu \mathrm{M}$ Picrotoxin and $1 \mu \mathrm{M}$ Strychnine). Hash symbol: significant difference between modulation of ON and OFF RGCs in a or between ON RGCs without and with pharmacological blockers in $c\left(p<10^{-4}\right.$, two-tailed Wilcoxon rank-sum test). 
Figure 5

a

Response to luminance steps alone

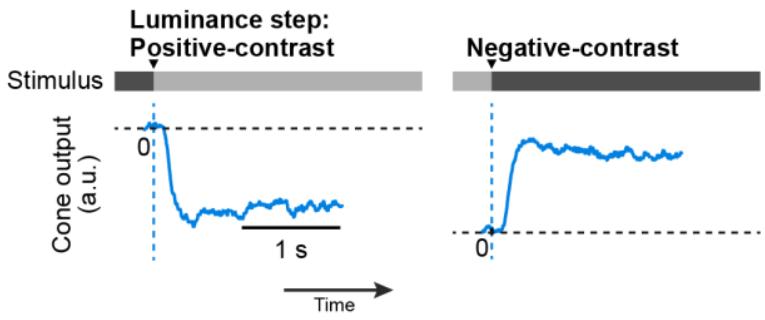

b Responses to luminance steps followed by probe flashes
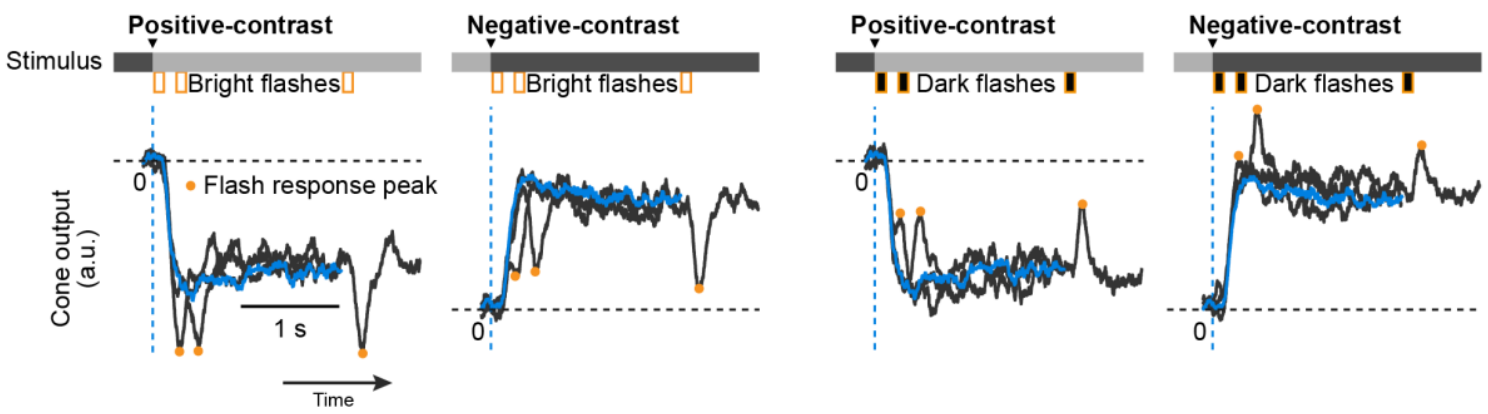

C Flash-induced responses
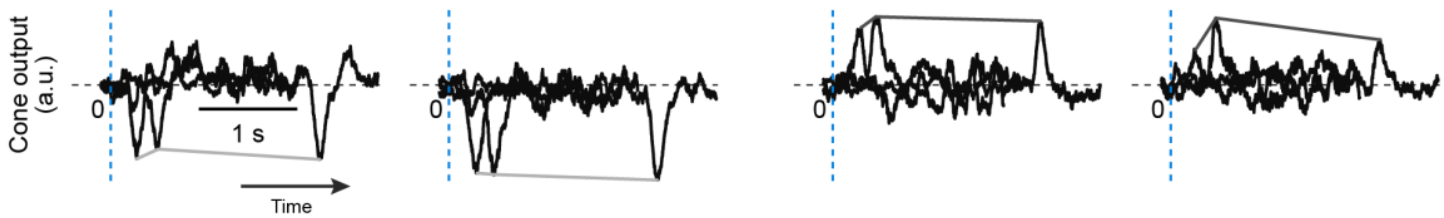

Figure 5 Cone output in response to probe flashes following luminance steps. a,b. Cone responses (baseline normalized iGluSnFR indicator fluorescence signal) to positive- and negative-contrast ( +0.4 and -0.4 on Michelson scale) luminance steps alone (a) and to luminance steps followed by probe flashes at 17, 250 and $2000 \mathrm{~ms}$ (orange bars below the intensity bar shows timing of probe flashes) (b). Probe flashes were either bright or dark (+0.33 or -0.33 Michelson contrast respectively; $100 \mathrm{~ms}$ long). In b, responses to step-alone (blue) and individual step $\rightarrow$ flash pairs (dark gray) are overlaid. Dashed blue lines: timing of luminance step; orange circles: peak cone response to flashes; horizontal dashed line: cone level prior to the luminance step. c. Flash-induced responses, isolated by subtracting luminance step alone responses (blue) from individual composite luminance step-probe flash responses (dark gray) in b. Lines connecting the response peaks highlight the time courses of suppression relative to baseline flash-induced $(2000 \mathrm{~ms})$ responses. 
bioRxiv preprint doi: https://doi.org/10.1101/2020.08.21.261198; this version posted September 10, 2020. The copyright holder for this preprint (which was not certified by peer review) is the author/funder, who has granted bioRxiv a license to display the preprint in perpetuity. It is made available under aCC-BY-NC-ND 4.0 International license.

Figure 6

a

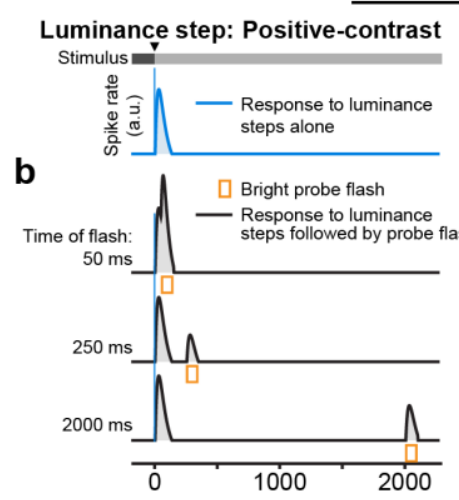

Model ON RGC

Negative-contrast
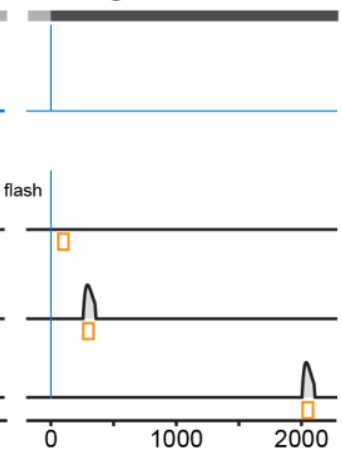

Model OFF RGC

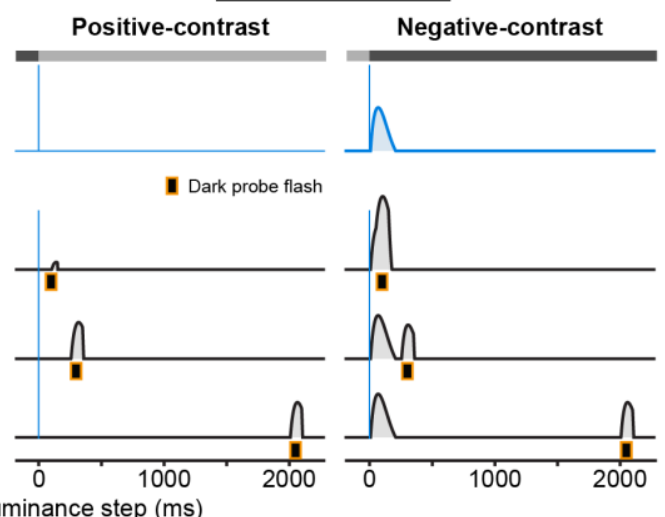

C
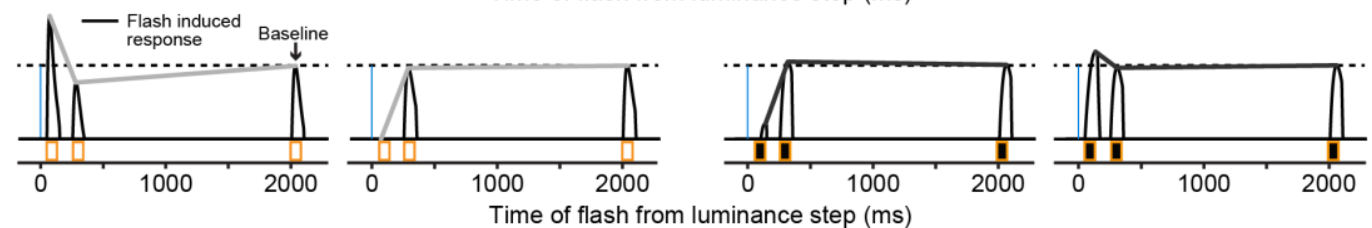

d
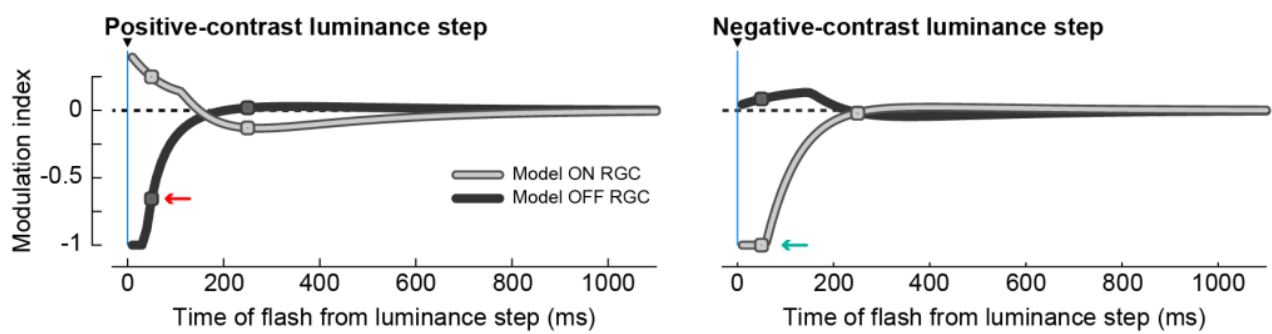

e

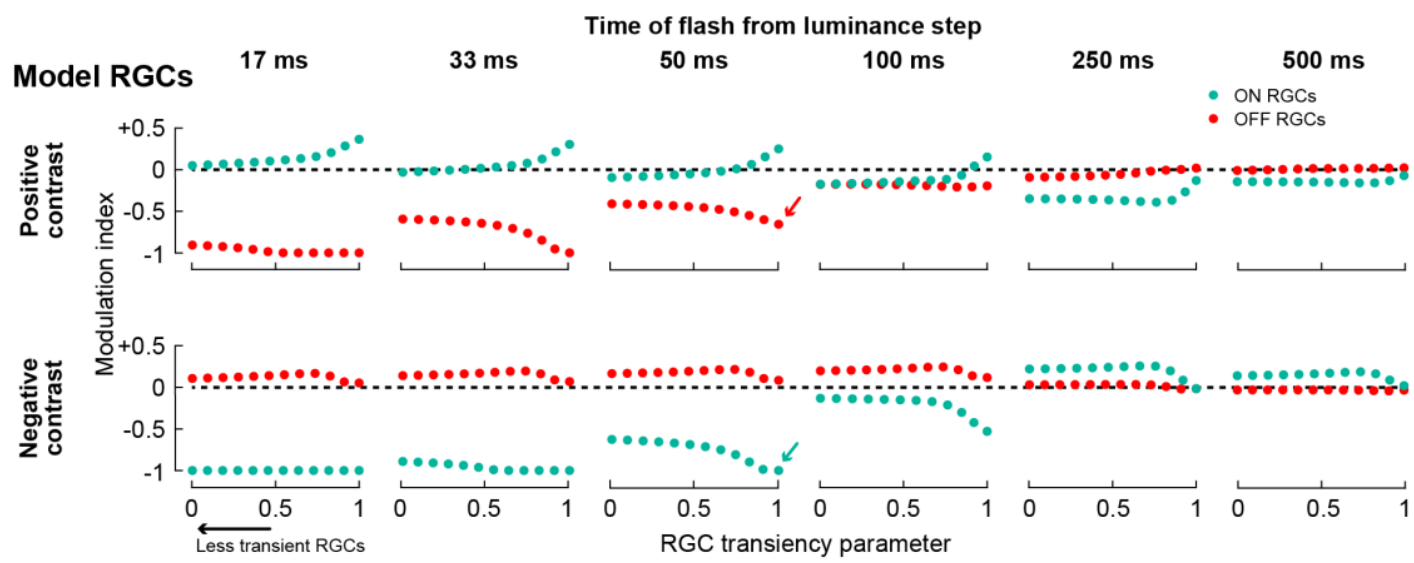

\section{Real RGCs}

雚
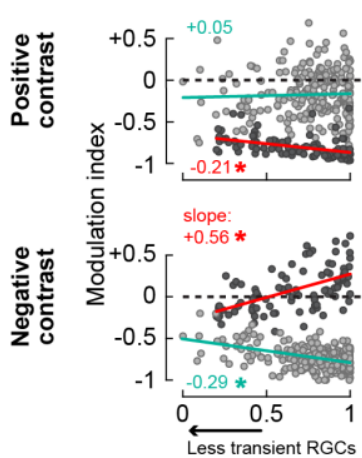

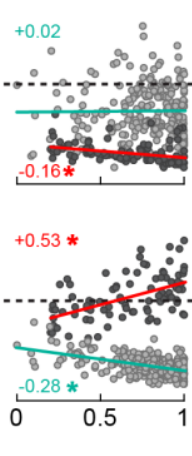

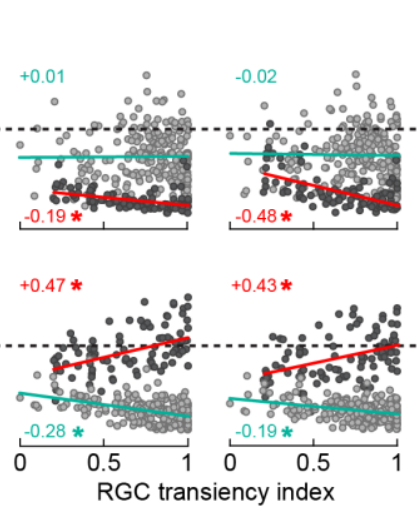

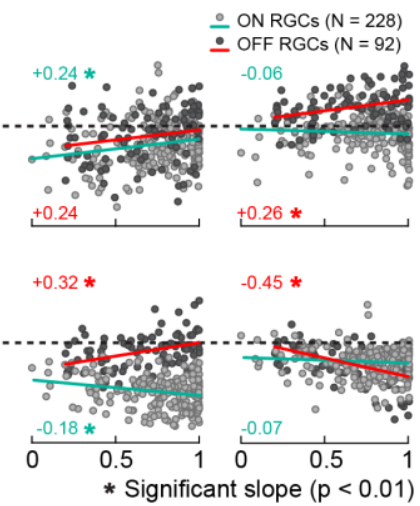


Figure 6 Model RGC responses to probe flashes following luminance steps. luminance steps alone (a, blue) and to luminance steps followed by probe flashes (b, black) at 50, 250 and 2000 ms (different rows; analogous to real RGCs in Fig. 1b, c). Luminance steps are depicted by intensity bars in a. First column in each cell type: responses following a positive-contrast luminance step; second column: responses following a negative-contrast luminance step. Vertical blue lines: timing of luminance step; orange bars: timing of probe flashes. Note the ON RGC and OFF RGC did not spike in response to negative-contrast (column 2) and positive-contrast (column 3 ) luminance steps respectively.

c. Flash-induced responses, after subtracting a from b, overlaid to show the modulation of probe flash responses at different times (analogous to real RGCs in Fig. 1d). Lines connecting the response peaks highlight the time courses of suppression relative to baseline flash-induced responses $(2000 \mathrm{~ms})$. gray), following positive-contrast (left panel) and negative-contrast (right panel) luminance steps. Modulation indices were calculated based on model responses to probe flashes presented at $10 \mathrm{~ms}$ intervals after luminance steps, and baseline as response to a flash at $2000 \mathrm{~ms}$. Circle markers indicate modulation indices based on probe flashes at 50 and $250 \mathrm{~ms}$ shown in b, c. Cyan and red arrows highlight the suppression of opposite-contrast flashes at $50 \mathrm{~ms}$ in ON and OFF RGCs, respectively. e. Modulation indices of model ON (cyan) and OFF (red) RGCs plotted as a function of RGC transiency. Individual panels correspond to different flash times (chosen from real-RGC recordings, Fig. 4) after positive-contrast (top row) and negative-contrast (bottom row) luminance steps. In a-d, transiency parameter was set to 1. Arrows highlight the same data as in $\mathbf{d}$.

812 f. Same as in e but for real ON (light gray circles; $N=228$; cyan line: linear regression 813 fit) and OFF RGCs (dark gray circles; $N=92$; red line: linear regression fit). These RGCs are a subset of the population data shown in Fig. $4 \mathrm{~b}$ for which we could compute a transiency index (Methods). Consistent with model predictions, suppression after negative-contrast steps was weaker in less transient ON RGCs (bottom row, blue regression line has negative slope) and, after positive-contrast steps, suppression was weaker in less transient OFF RGCs (top row, red regression line has negative slope). Numbers in each panel indicate the slope of the fits and asterisk symbol indicates statistically significant slope (slope $\neq 0, p<0.01$, two-tailed t-test). model cone responses. 
a
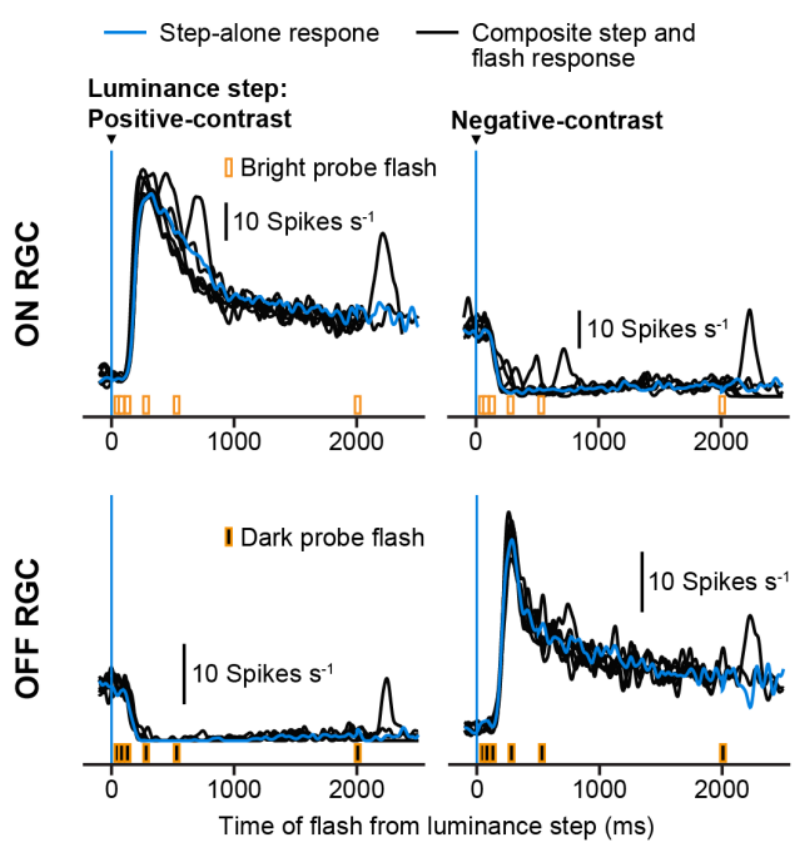

b

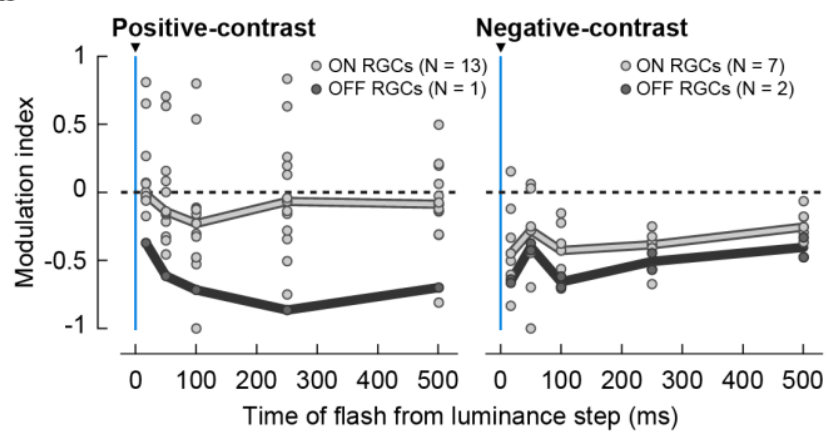

825 Figure 7 Retinal saccadic suppression in Macaque RGCs.

826 a. Average activity of an example macaque ON RGC (top) and OFF RGC (bottom) to positive-contrast (left column) and negative-contrast (right column) luminance steps alone (blue traces) and luminance steps followed by probe flashes (black traces). ON RGCs were analyzed for bright probe flashes and OFF RGCs for dark probe flashes.

830 Responses were averaged across the different positive-contrast (0.05 to 0.5 831 Michelson contrast, $\mathrm{N}=10$ sequences) and negative-contrast luminance steps (-0.05 832 to -0.5 Michelson contrast, $\mathrm{N}=10$ sequences).

833 b. Median modulation index (thick lines) of macaque ON (light gray) and OFF (dark gray) RGCs for probe flashes presented after positive-contrast (left panel; $N=13$ ON 835 RGCs, N = 1 OFF RGC) and negative-contrast luminance steps (right panel; $N=7$ ON 836 RGCs, $N=2$ OFF RGC). Circles represent modulation indices of individual RGCs. 837 This experiment was performed under scotopic light conditions. 
a

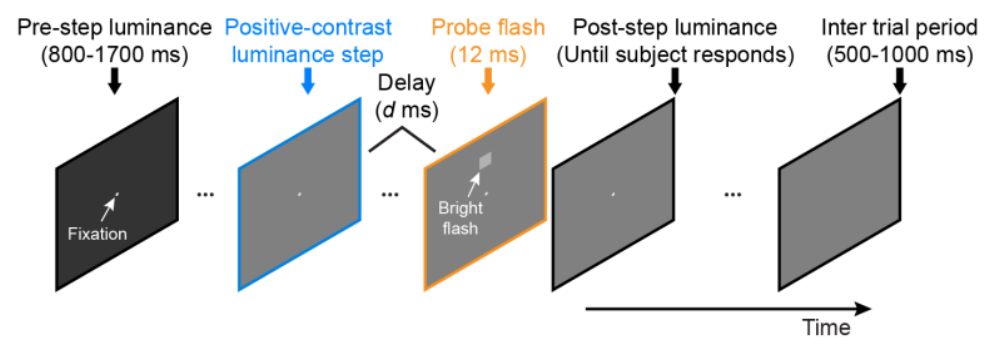

b
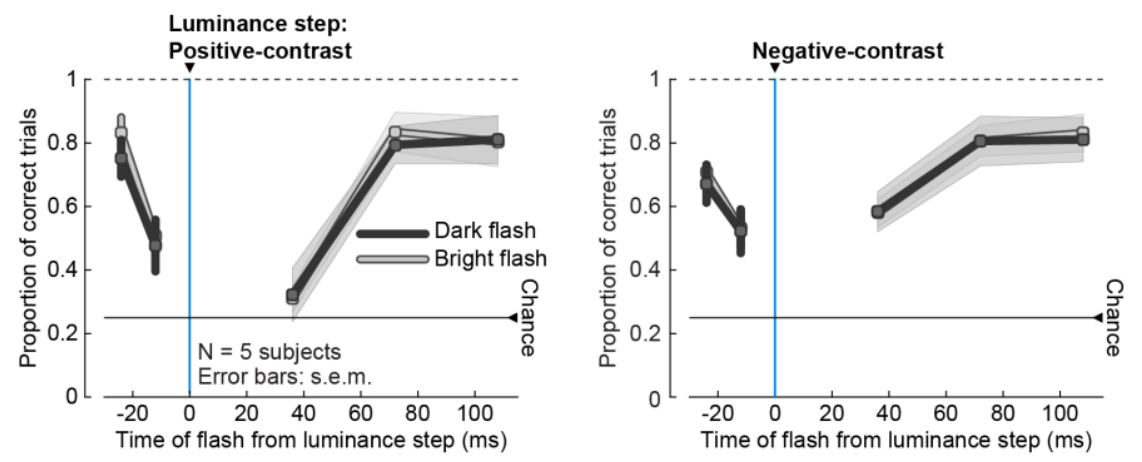

840 Figure 8 Perceptual suppression following luminance steps.

841 a. Example visual task trial. Subjects fixated a small spot on a uniform background with a set luminance (pre-step luminance) for a random duration (800-1700 ms). Background luminance then increased (like shown here) or decreased (positive or negative-contrast luminance step, respectively). At one of 5 times relative to the luminance step $(-24,-12,36,72$, or $108 \mathrm{~ms})$, a luminance pedestal (probe flash, $147.8 \times 147.8 \mathrm{~min}$ arc) was applied for $\sim 12 \mathrm{~ms}$ at one of four locations relative to the fixation spot: 7 deg above (shown here), below, to the right, or to the left. The probe flash was brighter (shown here) or darker than the current screen luminance. The background remained at the post-step luminance until the subject responded with the perceived location of the flash, plus an additional $500-1000 \mathrm{~ms}$ but without the fixation spot, allowing the subject to relax. The current luminance was the pre-step luminance of the consecutive trial.

b. Performance of human subjects (mean \pm s.e.m., $N=5$ subjects), to correctly localize a dark (dark gray) or a bright (light gray) probe flash presented at different times relative to positive-contrast (left panel) and negative-contrast (right panel) luminance steps (blue line). Each subject's responses were averaged across the different positive-contrast ( 0.3 to 0.56 Michelson contrast) and negative-contrast (-0.3 to -0.56 Michelson contrast) luminance steps. Perceptual performance was reduced around the time of luminance steps, reflecting suppression, irrespective of the combination of luminance step polarity and flash polarity. There were no statistically significant differences in suppression of dark and bright probe flashes (two-tailed Wilcoxon rank-sum test). Note that in the right panel, the suppression profile for bright probe flashes almost completely overlaps the suppression profile of dark probe flashes. 


\section{Experimental model and subject details}

867

868

\section{Animals}

We performed electrophysiological experiments on ex vivo mouse, pig and macaque retinae; and imaging experiments on ex vivo mouse retinae.

Mouse and pig ex vivo retinae experiments were performed in Tübingen, in accordance with German and European regulations, and animal experiments were approved by the Regierungspräsidium Tübingen. Macaque ex vivo retina experiment was performed at Stanford University. Eyes were removed from a terminally anesthetized macaque rhesus monkey used by other laboratories in the course of their experiments, in accordance with the Institutional Animal Care and Use Committee guidelines of Stanford University.

For mouse retina electrophysiology, we used 47 retinae from 15 male and 30 female

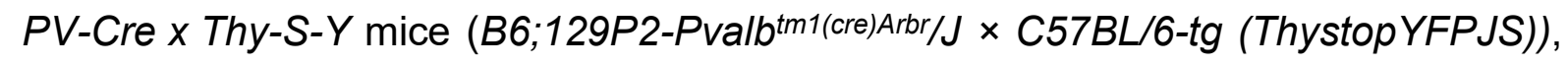
3-12 months old, which are functionally wild type (Farrow et al., 2013; Münch et al., 2009; Tikidji-Hamburyan et al., 2015). Additionally, we recorded the cone output from 4 retinae obtained from two C57BL/6 male mice, 9 to 10 weeks old. We housed mice on a $12 / 12 \mathrm{~h}$ light/dark cycle, in ambient temperatures between $20-22{ }^{\circ} \mathrm{C}$ and humidity levels of $40 \%$.

We also replicated experiments on 9 pig retinae obtained from domestic female pigs after they had been sacrificed during independent studies at the Department of Experimental Surgery at the Medical Faculty of the University of Tübingen. Pigs were anesthetized using atropine, azaperone, benzodiazepine (midazolam), and ketamine, and then sacrificed with embutramide (T61). Before embutramide administration, heparin was injected.

One experiment was conducted with a retina extracted from a macaque rhesus monkey. 


\section{Humans}

Human psychophysics experiments were performed in Tübingen. Human subjects provided written, informed consent, and they were paid 10 Euros per session of 60 minutes each, for three sessions. Human experiments were approved by ethics committees at the Medical Faculty of Tübingen University, and they were in accordance with the Declaration of Helsinki.

In total, we collected data from 5 subjects (24-29 years old; one female).

\section{Experimental setup}

\section{Retina electrophysiology (mouse and pig): procedure and laboratory setup}

Mice were dark adapted for 4-16 $\mathrm{h}$ before experiments. We then sacrificed them under dim red light, removed the eyes, and placed eyecups in Ringer solution (in mM: 110 $\mathrm{NaCl}, 2.5 \mathrm{KCl}, 1 \mathrm{CaCl}_{2}, 1.6 \mathrm{MgCl}_{2}, 10 \mathrm{D}$-glucose, and $22 \mathrm{NaHCO}_{3}$ ) bubbled with $5 \%$ $\mathrm{CO}_{2}$ and $95 \% \mathrm{O}_{2}$. We removed the retina from the pigment epithelium and sclera while in Ringer solution.

Pigs were dark-adapted for 15-20 $\min$ before sacrifice. Immediately after veterinary-confirmed sacrifice, the eyes were enucleated under dim red light, and the cornea, lens, and vitreous were removed. Eyecups were kept in $\mathrm{CO}_{2}$-independent culture medium (Gibco) and protected from light. We transported eyecups to our laboratory and cut pieces from mid-peripheral or peripheral retinae. Only those retinae which showed ganglion cell responses to light stimuli were used in our experiments.

We recorded mouse and pig retinal ganglion cell (RGC) activity using either low- or high-density multi-electrode arrays (MEAs). The low-density setup consisted of a perforated 60-electrode MEA (60pMEA200/30ir-Ti-gt, Multichannel Systems (MCS), Reutlingen, Germany) having a square grid arrangement and $200 \mu \mathrm{m}$ inter-electrode distance. We whole mounted an isolated retina on a nitrocellulose filter (Millipore) with a central $2 \times 2 \mathrm{~mm}$ hole. The mounted retina was placed with the RGC side down into the recording chamber, and good electrode contact was achieved by negative pressure through the MEA perforation. We superfused the tissue with Ringer solution at $30-34{ }^{\circ} \mathrm{C}$ during recordings, and we recorded extracellular activity at $25 \mathrm{kHz}$ using 
a USB-MEA-system (USB-MEA 1060, Multichannel Systems) or a memory-card based system (MEA1060, Multichannel Systems). Data was acquired using MC Rack version 4.6.2 (Multichannel Systems). A detailed step-by-step approach is provided in (Reinhard et al., 2014).

The high-density MEA setup consisted of either a HiDens CMOS MEA (Frey et al., 2009) (developed by the lab of Andreas Hierlemann, Basel, Switzerland) or a MaxOne system (Müller et al., 2015) (Maxwell Biosystems, Basel, Switzerland). The HiDens CMOS MEA featured 11,011 metal electrodes with inter-electrode (center-to-center) spacing of $18 \mu \mathrm{m}$ placed in a honeycomb pattern over an area of $2 \times 1.75 \mathrm{~mm}$. Any combination of 126 electrodes could be selected for simultaneous recording. The MaxOne MEA featured 26,400 metal electrodes with center-to-center spacing of 17.5 $\mu \mathrm{m}$ in a grid-like arrangement over an area of $3.85 \times 2.1 \mathrm{~mm}$. In this system, up to 1024 electrodes could be selected for simultaneous recordings. For each experiment, a piece of isolated retina covering almost the entire electrode array was cut and placed RGC-side down in the recording chamber. We achieved good electrode contact by applying pressure on the photoreceptor side of the retina by carefully lowering a transparent permeable membrane (Corning Transwell polyester membrane, $10 \mu \mathrm{m}$ thick, $0.4 \mu \mathrm{m}$ pore diameter) with the aid of a micromanipulator. The membrane was drilled with $200 \mu \mathrm{m}$ holes, with center-center distance of $400 \mu \mathrm{m}$, to improve access of the Ringer solution to the retina. We recorded extracellular activity at $20 \mathrm{kHz}$ using FPGA signal processing hardware. In the case of the HiDens CMOS MEA, data were acquired using custom data acquisition software, called MEA 1k Scope (developed by the lab of Andreas Hierlemann, Basel, Switzerland). In the case of the MaxOne MEA, data were acquired using MaxLab software provided by Maxwell Biosystems, Basel, Switzerland.

In total, we performed 59 recordings, 47 from mouse and 12 from pig retinae. 24 of the 59 recordings were done using low-density MEAs. Once a basic experimental protocol was established, we shifted to HiDens CMOS MEA providing much higher 949 throughput. 12 experiments were done using this setup. We upgraded to the MaxOne 950 MEA for even higher throughput and did 23 recordings using this setup. A subset of the data collected from 32 of the 59 recordings (20 from mouse and 12 from pig 
retinae), was also used in our previous study (Idrees et al., 2020). Here, we show further in-depth analysis of that data.

We presented light stimuli to the retinal piece that was placed on the MEA using a DLP projector running at $60 \mathrm{~Hz}$ (Acer K11 for low-density MEA experiments and Lightcrafter 4500 from EKB Technologies Ltd. with internal red, green and blue light-emitting diodes, for high-density MEA experiments). $60 \mathrm{~Hz}$ is above the flicker fusion frequency of both mouse and pig retinae; therefore, the framerate of these projectors was adequate for our purposes. The Acer K11 projector had a resolution of $800 \times 600$ pixels covering $3 \times 2.25 \mathrm{~mm}$ on the retinal surface. Lightcrafter 4500 had a resolution of $1280 \times 800$ pixels, extending $3.072 \times 1.92 \mathrm{~mm}$ on the retinal surface. We focused images onto the photoreceptors using a condenser (low-density MEA recordings, illumination from below) or a $5 x$ objective (high-density MEAs, illumination from above). In each case, the light path contained a shutter and two motorized filter wheels with a set of neutral density (ND) filters (Thorlabs NE10B-A to NE50B-A), having optical densities from 1 (ND1) to 5 (ND5). The filters allowed us to adjust the absolute light level of the stimulation.

We measured the spectral intensity profile (in $\mu \mathrm{W} \mathrm{cm}{ }^{-2} \mathrm{~nm}^{-1}$ ) of our light stimuli with a calibrated USB2000+ spectrophotometer (Ocean Optics) and converted the physical intensity into a biological equivalent of photoisomerizations per rod photoreceptor per second $\left(R^{*} \operatorname{rod}^{-1} s^{-1}\right)$, as described before (Tikidji-Hamburyan et al., 2015). Light intensities of the projector output covered a range of 3 log units (i.e. 1000-fold difference between black and white pixels, over an 8-bit range). We linearized the projector output, and we used only grayscale images of limited contrast, spanning at most the range from 0 to 120 in the 8-bit range of the projector (see stimulus description below for details). Absolute light intensities were set to the mesopic level, where a stimulus intensity of ' 30 ' in our 8-bit DLP projector scale (0-255) corresponded to 225 to $1000 \mathrm{R}^{*} \mathrm{rod}^{-1} \mathrm{~s}^{-1}$, depending on the experimental rig used for the experiment (i.e. different DLP projectors and MEAs). We pooled all data from the different rigs as separate individual analyses from the individual setups revealed no effects of recording conditions in the different setups. For experiments of Fig. S4, we also recorded at scotopic light levels where a stimulus intensity of '30', corresponded to 23 $\mathrm{R}^{*} \operatorname{rod}^{-1} \mathrm{~s}^{-1}$ at scotopic level. 


\section{Retina electrophysiology (macaque): procedure and laboratory setup}

985

986

987

988

989

990

991

992

993

994

995

996

997

998

999

1000

1001

1002

1003

1004

1005

1006

In one experiment, we recorded the activity of macaque retinal ganglion cells. For this experiment we used a high-density MEA, as described previously (Chichilnisky and Baylor, 1999; Field et al., 2007). Following enucleation, the anterior portion of the eye and vitreous were removed. The eye was stored in a dark container in oxygenated Ames' solution (Sigma, St. Louis, $\mathrm{MO}$ ) at $33^{\circ} \mathrm{C}, \mathrm{pH}$ 7.4. Under infrared illumination, a small piece of retina approximately $1 \times 1 \mathrm{~mm}$, from a retinal region with eccentricity around $12 \mathrm{~mm}$ (4.0-17mm temporal equivalent eccentricity; (Chichilnisky and Kalmar, 2002)), was dissected and placed ganglion cell side down on a MEA for recording. The retina pigment epithelium remained attached during the recording; the retina was perfused with oxygenated Ames' solution. A custom planar large-scale MEA (Field et al., 2007; Litke et al., 2004) with a hexagonal outline of 519 electrodes at $30 \mu \mathrm{m}$ pitch was used. Recorded voltages were band-pass filtered, amplified, and digitized at 20 $\mathrm{kHz}$ using custom electronics (Litke et al., 2004). Spike sorting process was described previously (Field et al., 2007).

Visual stimulation was performed with the optically reduced image of a gamma-corrected OLED microdisplay (eMagin) refreshing at $60.35 \mathrm{~Hz}$ focused on the photoreceptor outer segments. The visual stimulus was delivered through the mostly-transparent electrode array. The power of each display primary was measured at the preparation with a calibrated photodiode (UDT Instruments). At the mean background illumination level, the photoisomerization rates for the rods and the $L, M$, and $\mathrm{S}$ cones were approximately $29,9,9$, and $2 \mathrm{P}^{*}$ receptor ${ }^{-1} \mathrm{~S}^{-1}$, respectively (see $\mathrm{Li}$ et al., 2014), placing the retina in scotopic regime.

\section{Retina electrophysiology: pharmacology}

In several MEA experiments, we used pharmacological agents to block specific receptors in the mouse retina. To block $\mathrm{GABA}_{\mathrm{A}}$ receptors selectively, we used $5 \mu \mathrm{M}$ SR-95531 (gabazine, an antagonist of GABAA receptors; Sigma). To block both $\mathrm{GABA}_{A}$ and GABAc receptors, we used $100 \mu \mathrm{M}$ picrotoxin (an antagonist of $\mathrm{GABA}_{A}$ and GABAc receptors; Sigma). To block glycine receptors, we used $1 \mu \mathrm{M}$ Strychnine (antagonist of Glycine receptors). 
We first prepared a 1000x stock solution of these pharmacological blockers as follows: SR-95531 was dissolved in water at a concentration of $5 \mathrm{mM}$; picrotoxin was dissolved in DMSO at a concentration of $100 \mathrm{mM}$; Strychnine was dissolved in Chloroform at a concentration of $1 \mathrm{mM}$. During the experiments, we pipetted the stock solution to the Ringer solution in a 1:1000 ratio. Wash-in was performed for 20 min.

\section{Cone photoreceptors imaging: procedure and laboratory setup}

To record the output of cone photoreceptors in the mouse retina, we measured the glutamate release using an intensity based glutamate-sensitive fluorescent reporter, iGluSnFR (Marvin et al., 2013) expressed in horizontal cell processes post-synaptic to cone terminals, using a viral approach. We recorded the cone output from 4 retinae obtained from two C57BL/6 male mice, 9 to 10 weeks old. Below, we reproduce the methods, previously described in Szatko et al (Szatko et al., 2020).

We dark-adapted the mice for $\geq 1 \mathrm{~h}$ before the experiments. They were then anaesthetized using isoflurane (Baxter) and sacrificed by cervical dislocation. The eyes were enucleated and hemisected in carboxygenated $\left(95 \% \mathrm{O}_{2}\right.$ and $\left.5 \% \mathrm{CO}_{2}\right)$ artificial cerebrospinal fluid (ACSF) solution containing (in $\mathrm{mM}$ ): $125 \mathrm{NaCl}, 2.5 \mathrm{KCl}, 2$ $\mathrm{CaCl}_{2}, 1 \mathrm{MgCl}_{2}, 1.25 \mathrm{NaH}_{2} \mathrm{PO}_{4}, 26 \mathrm{NaHCO}_{3}, 20$ glucose, and 0.5 L-glutamine (pH 7.4). We then moved the tissue to the recording chamber where it was continuously perfused with carboxygenated ACSF at $\sim 36{ }^{\circ} \mathrm{C}$. In these experiments, ACSF contained $\sim 0.1 \mu \mathrm{M}$ Sulforhodamine-101 (SR101, Invitrogen) to reveal blood vessels and any damaged cells in the red fluorescence channel (Euler et al., 2019). All procedures were carried out under very dim red (>650 nm) light.

iGluSnFR was expressed in the retina by viral transduction of AAV2.7m8.hSyn.iGluSnFR, generated in the Dalkara lab (Institut de la Vision) as described in (Dalkara et al., 2013; Khabou et al., 2016). The iGluSnFR plasmid construct was provided by J. Marvin and L. Looger (Janelia Research Campus, USA). A volume of $1 \mu \mathrm{L}$ of the viral construct was injected into the vitreous humour of the mice, anaesthetized with 10\% Ketamine (Bela-Pharm GmbH \& Co. KG) and 2\% xylazine (Rompun, Bayer Vital $\mathrm{GmbH}$ ) in $0.9 \% \mathrm{NaCl}$ (Fresenius). For the injections, we used a micromanipulator (World Precision Instruments) and a Hamilton injection 
system (syringe: 7634-01, needles: 207434, point style 3, length $51 \mathrm{~mm}$, Hamilton Messtechnik $\mathrm{GmbH}$ ). Imaging experiments were performed 3-4 weeks after injection. In the outer retina, iGluSnFR was predominantly expressed in horizontal cells. As the expression tended to be weaker in the central retina, most scan fields were acquired in the medial to peripheral ventral or dorsal retina.

To record the iGluSnFR signal, we used a MOM-type two-photon microscope setup (designed by W. Denk, MPI, Heidelberg; purchased from Sutter Instruments / Science Products). The design and procedures have been previously described in (Euler et al., 2009, 2019). In brief, the system was equipped with a mode-locked Ti:Sapphire laser (MaiTai-HP DeepSee, Newport Spectra-Physics), two fluorescence detection channels for iGluSnFR (HQ 510/84, AHF/Chroma) and SR101 (HQ 630/60, AHF), and a water immersion objective (W Plan-Apochromat 20x /1.0 DIC M27, Zeiss). The laser was tuned to $927 \mathrm{~nm}$ for imaging iGluSnFR. For image acquisition, we used custom made software (ScanM by M. Müller and T. Euler) running under IGOR Pro 6.3 for Windows (Wavemetrics), taking time-lapsed $128 \times 128$ pixel image scans at $3.9 \mathrm{~Hz}$ in the outer plexiform layer (OPL).

For light stimulation in cone imaging experiments, we used the Lightcrafter $(\mathrm{LCr}$; DPM-E4500UVBGMKII), a DLP projector from EKB Technologies Ltd. with internal UV and green light-emitting diodes (LEDs). The light from the DLP projector was focused through the objective. To optimize spectral separation of mouse $\mathrm{M}$ - and $\mathrm{S}$ - opsins, LEDs were band-pass filtered (390/576 Dualband, F59-003, AHF/Chroma). LEDs of the DLP projector were synchronized with the microscope's scan retrace. Stimulus intensity (as isomerization rate, $\mathrm{P}^{*} \mathrm{cone}^{-1} \mathrm{~s}^{-1}$ ) was calibrated to range from $\sim 500$ (black image) to $\sim 20,000$ for M- and S-opsins. In addition, a steady illumination component of $\sim 10^{4} \mathrm{P}^{*} \mathrm{cone}^{-1} \mathrm{~s}^{-1}$ was present during the recordings because of two-photon excitation of photopigments. The overall light intensity falling on to the retina was therefore in the low photopic regime. The light stimulus was centered to the recording field before every experiment. For all experiments, the retinal tissue was kept at a constant mean stimulator intensity level for at least $15 \mathrm{~s}$ after the laser scanning started and before stimuli were presented. 


\section{Human psychophysics: laboratory setup}

We used a similar laboratory setup as described previously (Idrees et al., 2020). Briefly, subjects sat in a dark room $57 \mathrm{~cm}$ in front of a CRT monitor ( $85 \mathrm{~Hz}$ refresh rate; 41 pixels per deg resolution) spanning $34.1 \times 25.6$ deg (horizontal $x$ vertical). Head fixation was achieved with a custom head, forehead, and chin rest (Hafed, 2013), and we tracked eye movements of the left eye at $1 \mathrm{kHz}$ using a video-based eye tracker (EyeLink 1000, SR Research Ltd, Canada). Gray backgrounds in the luminance step experiment (Fig. 8) were always presented at an average luminance of $49.84 \mathrm{~cd} \mathrm{~m}^{-2}$, and the monitor was linearized (8-bit resolution) such that equal luminance increments and decrements for luminance steps were possible around this average. In total, we collected data from 5 subjects (24-29 years old; one female). A subset of the data from 4 subjects was used in our previous study (Idrees et al., 2020). Here, we perform novel analyses of the complete dataset, in addition to one new subject.

\section{Visual stimuli: Retina electrophysiology (Figs. 1-4, 7, S3-S10, S13)}

In retina electrophysiology experiments, we used two broad visual stimulation paradigms: a saccade (texture displacements) paradigm (Fig. S1a), and a luminance step paradigm (Fig. S1b), described in detail below. In different experiments we used different spatial and/or pharmacological manipulations of these two paradigms.

\section{Saccade (texture displacements) paradigm}

Background textures

We created background textures (Fig. S2a) by convolving a random binary (i.e. white or black) pixel image with a two-dimensional Gaussian blurring filter (Schwartz et al., 2012) defined by the kernel

$$
G(x, y)=e^{\frac{-\left(x^{2}+y^{2}\right)}{2 \sigma^{2}}}
$$

The parameter $\sigma$ of the kernel influenced the amount of blurring. This resulted in textures having effectively low-pass spectral content (Fig. S2b) with a cutoff frequency depending on $\sigma$. For easier interpretation, we define the spectral content of these textures by a spatial scale. Intuitively, the spatial scale approximates the size of the 
smallest dark and bright image blobs of the texture (Fig. S2a). Quantitatively, the spatial scale is defined as the $2 * \sigma$ parameter of the Gaussian blurring filter. We generated textures with four different spatial scales: 25, 50, 150 and $300 \mu \mathrm{m}$, that resulted in dark and bright image blobs approximating a range of receptive field sizes between bipolar cells (texture with spatial scale $25 \mu \mathrm{m}$, see (Zhang et al., 2012)) and RGCs (textures with spatial scale 150 and $300 \mu \mathrm{m}$ ). In other words, coarser textures matched the resolution of RGCs, and finer textures matched the resolution of one processing stage earlier, the retinal bipolar cells. Calculating power spectra for the textures (Fig. S2b) confirmed that the spatial scale and hence the cutoff frequencies were consistent with this design aim. In different experiments, we used textures of all or a subset of the different spatial scales.

We normalized the pixel intensities in the textures to have uniform variations in luminance around a given mean. We used pixel intensities (from our 8-bit resolution scale) ranging from 0 to 60 around a mean of 30 , or ranging from 30 to 90 around a mean of 60 (see sub-section Saccades and probe flashes for when each paradigm was used).

\section{Saccades and probe flashes}

To simulate saccades in our ex vivo retina electrophysiology experiments, we displaced the texture across the retina in 6 display frames $(100 \mathrm{~ms}$ at $60 \mathrm{~Hz}$ refresh rate). For easier readability, we usually refer to these saccade-like texture displacements as "saccades". The textures were displaced in each frame by a constant distance along a linear trajectory. While each "saccade" lasted $100 \mathrm{~ms}$, displacement direction was varied randomly for each "saccade" (uniformly distributed across all possible directions), and "saccade" amplitude could range from $310 \mu \mathrm{m}$ to $930 \mu \mathrm{m}$ (corresponding to a velocity range of $3100-9300 \mu \mathrm{m} \mathrm{s}^{-1}$ on the retinal surface). In visual degrees, this corresponds to a velocity range of $100-300 \mathrm{deg} \mathrm{s}^{-1}$ and displacement range of 10-30 deg in mice, well in the range of observed mouse saccade amplitudes (Sakatani and Isa, 2007). Similar to primates, mice also have oculomotor behavior, even under cortical control (Itokazu et al., 2018). For example, they make, on average, 7.5 saccade-like rapid eye movements per minute when their head is fixed (Sakatani and Isa, 2007) (humans make several saccades per second). 
We used the same retinal displacement range of $310 \mu \mathrm{m}$ to $930 \mu \mathrm{m}$ for pig retinae. To the best of our knowledge, pig oculomotor behavior has not been documented in the literature. However, with their larger eyeball sizes, our translations of the retinal image would correspond to slower saccades (e.g. small saccades in humans and monkeys), which are also associated with saccadic suppression. Moreover, retinal "saccadic suppression" is not critically dependent on the details of movement kinematics, as it is triggered by visual transients (Fig. 4, also see Figs. 4, 5 in Idrees et al., 2020).

Each trial consisted of successive sequences (Fig. 1a, S1a) that combined a "saccade" with a probe flash, as follows: there was first a "pre-saccade" fixation of 2 seconds, where the texture remained static over the retina, then a $100 \mathrm{~ms}$ "saccade", followed by "post-saccade" fixation where the texture again remained static over the retina but now with a shifted texture. At a certain time from "saccade" onset (delay $d$, range: $50 \mathrm{~ms}$ to $2100 \mathrm{~ms}$ ), we presented a probe flash (see below). Following the probe flash, the texture remained static at the post-saccade fixation position for another 2 seconds before the next saccade of the successive sequence occurred. The post-probe-flash fixation of one sequence was therefore also the pre-saccade fixation of the next sequence. This way the texture remained visible during the entire trial, being translated during saccades of the successive sequences. In a single trial, 39 such sequences occurred. In each successive sequence, the direction and amplitude of the saccade was pseudo-randomly determined by the range of allowed saccade amplitudes and directions. The texture always landed at unique locations within a trial. The end result was that, within a single trial, RGCs experienced a wide spectrum of saccade amplitudes, directions and contrasts across these 39 saccades. As such, by analyzing the average effects of the 39 saccades on RGC responses to probe flashes, we captured a wide range of saccade-induced kinematics and luminance changes over the RGC receptive fields.

In most cases, the probe flash had a duration of 2 frames ( $\sim 33 \mathrm{~ms})$. We used 1 frame ( $\sim 16 \mathrm{~ms})$ in a subset of earlier experiments (mouse: 709 of 1616 cells; pig: 116 of 228 cells). Results were pooled across these paradigms as the effects were indistinguishable. The probe flash was a full-screen positive ("bright") or negative (“dark") stimulus transient. 
Bright or dark probe flashes could happen in two different ways across our experiments. The results were indistinguishable between the two ways, so we pooled results across them. Briefly, in one manipulation, the probe flash replaced the texture with a homogeneous bright (pixel intensity of 60 in our 8-bit projectors) or dark (pixel intensity of 0 ) full-screen (in these experiments, the textures themselves had intensities ranging from 0 to 60 pixel intensity; see Background textures above). This way, the flash contrast from the underlying background luminance was variable across space (e.g. a bright flash on a bright portion of a texture had lower contrast from the underlying texture than the same flash over a dark portion of the texture). In the second manipulation, the bright and dark flashes were simply luminance increments or decrements (by pixel values of 30 on our 8-bit projectors) over the existing textures. This way, spatial contrast relationships in the background textures were maintained. In these experiments, the textures themselves had a range of 30-90 pixel intensities and a mean pixel value of 60 (on our 8-bit projectors). Out of the 1616 RGCs that we analyzed for saccadic suppression across all experiments where texture displacements were used as saccades (irrespective of the spatial or pharmacological manipulations), 1129 RGCs experienced such probe flashes, whereas the rest (487 RGCs) experienced the homogenous probe flash. For pig retina recordings, we always used the homogenous framework. However, in the subset of pig experiments where the 2-frame probe flash was employed (112 of 228 RGCs), we used a high-contrast probe flash such that a bright flash would be achieved by first going to 0 in the first frame of the flash then going to 60 (on our 8-bit projectors) in the next frame (and vice versa for a dark flash). Again, all data were pooled across these different paradigms because their outcomes were indistinguishable.

The number of trials required during a physiology experiment depended on the number of conditions that we ran on a specific day. For example, testing 7 different flash delays required 15 trials ( 7 with bright probe flashes, 7 with dark probe flashes, and 1 without probes). In a given experiment, we always interleaved all the conditions; that is, in any one of the 15 necessary trials, each of the 39 saccades could be followed by a bright or a dark probe at any of the 7 delays, or no probe flash at all (Fig. S1a shows schematic of one such trial). Moreover, we repeated the total number of conditions (e.g. the interleaved 15 trials) 4 times per session, and we averaged responses across 
those repetitions. Since one trial typically lasted for 2 minutes, the example of 15 trials repeated 4 times lasted for approximately 2 hours. This was usually combined with additional conditions (e.g. other background texture scales). Therefore, the total number of saccades shown in any given experiment could be computed by \#trials $\times 39$ saccades per trial $x$ \#textures $x$ \#repetitions. A typical experiment lasted 10-12 hours. If the combination of conditions would have required even longer recordings in a given experiment, we reduced the number of conditions (e.g. we presented flashes at fewer delays, or used fewer texture scales).

\section{Full-field saccades}

In the full-field saccades experiments, saccades and probe flashes occurred over the entire retina. This was our main experimental paradigm that we used for characterizing how saccades modulate RGC responses to probe flashes. This paradigm was also used as a control in experiments in which we applied different spatial or pharmacological manipulations of this paradigm to probe for the spatial origins and mechanisms of saccadic suppression. Further, results from this paradigm served as a baseline standard across different experimental rigs. This paradigm was used in a total of 32 retinal recordings (32 retinae from 30 mice). In different recordings, we used a subset of the texture scales and probe flash delays. This explains the different values of $\mathrm{N}$ seen for different conditions in, for example, Figs. 1e, S3. However, to ensure comparison, some conditions always overlapped across different recordings. This paradigm was also used in 12 recordings with retinae from 6 pigs.

\section{Periphery saccades (global component of suppression)}

In this manipulation, we restricted saccades to the RGC's receptive fields periphery (i.e. its far surround). This spatial manipulation was used to investigate the spatial origins of the global component of suppression (Figs. 2a-b, S5). We performed 13 recordings (13 retinae from 13 mice) with this paradigm, always with the high-density multielectrode array system (MaxOne by MaxWell), as it provided a large electrode area $\left(\sim 2 \times 4 \mathrm{~mm}^{2}\right)$ for the retina to be placed on. The recording region was typically either a high density block of electrodes (inter-electrode spacing: $\sim 17.5 \mu \mathrm{m}$ ) or a block with one-electrode spacing (inter-electrode spacing: $\sim 35 \mu \mathrm{m}$ ). The recording region was selected close to the center of the electrode array. We centered a large square mask $\left(1000 \times 1000 \mu \mathrm{m}^{2}\right)$ over the recording region to restrict the texture and saccade 
presentation to the periphery of RGC receptive fields (Fig. S5b). The mask had a homogenous intensity corresponding to the mean luminance of the texture. At different times relative to texture displacements, full-field probe flashes were presented, similar to experiments with full-field texture displacements. The intensity of each pixel of the stimulus (both the mask and the texture regions) was adjusted for the probe flashes, either decreased or increased by a pixel value of 30 (on our 8-bit projectors) for dark and bright probe flashes, respectively. In all periphery saccade experiments, we used probe flash duration of $\sim 33 \mathrm{~ms}$, and a coarse texture background of spatial scale 300 $\mu \mathrm{m}$.

\section{Checkerboard mask paradigm (local component of suppression)}

In this spatial manipulation we presented saccades and flashes in small square regions spread equidistantly over the entire retina. Each square region measured $100 \times 100 \mu \mathrm{m}^{2}$, separated from adjacent squares by an edge-edge gap of $100 \mu \mathrm{m}$. The gap was kept at mean luminance throughout the experiment. Saccades and flashes could either be presented in all the regions (similar to full-field saccades, except for the gap), or in alternate regions (Fig. 2e, S7a), arranged like in a checkerboard. This paradigm was used to investigate the origins of the local component of suppression (Figs. $2 \mathrm{f}, \mathrm{S} 7 \mathrm{a}-\mathrm{C}$ ). We performed 4 recordings (4 retinae from 3 mice) with this paradigm, always with the low density MCS MEA rig. In all experiments with this spatial manipulation, we used probe flash duration of $\sim 17 \mathrm{~ms}$, and a coarse texture background of spatial scale of $150 \mu \mathrm{m}$.

\section{Luminance step paradigm}

1251

In this paradigm (Fig. S1b), we used no textures at all. The screen was always a homogenous gray field, and the visual event of a "saccade" was replaced by an instantaneous step to a different gray value. The gray backgrounds had intensities between 30 and 90 (on our 8-bit projector). The instantaneous step in intensity caused either a positive contrast luminance step (in the range of +0.03 to +0.50 Michelson contrast) or a negative contrast luminance step ( -0.03 to -0.50 Michelson contrast). This paradigm was used to characterize the stimulus-stimulus interactions that ultimately trigger retinal saccadic suppression (Figs. 4, 7, S8-10). We performed a total of 4 recordings ( 4 retinae from 4 mice) with this paradigm, always with the high density 
1261

1262

1263

1264

1265

1266

1267

1268

1269

1270

1271

1272

1273

1274

1275

1276

1277

1278

1279

1280

1281

1282

1283

1284

1285

1286

1287

1288

1289

1290

1291

1292

(Fig. S1b) that each combined a luminance step with a probe flash, as follows: there was first a "pre-step" fixation of 2 seconds where the retina was exposed to a fixed gray level (analogous to "pre-saccade" fixation in texture displacements), then an instantaneous switch to "post-step" fixation (analogous to "post-saccade"). At a certain time from the luminance step (delay: 17, 33, 50, 100, 250, 500, 1000 or $2000 \mathrm{~ms}$ ), we presented a 2-frame ( $\sim 33 \mathrm{~ms})$ dark (-0.33 Michelson contrast) or bright $(+0.33$ Michelson contrast) probe flash. Some sequences contained no probe flash, the next luminance step then happened 4 seconds after the previous one. In a given experiment, we had 17 trials representing the 17 conditions: 8 flash delays $\times 2$ probe flash polarities +1 condition with no probe flash. Similar to the saccade paradigm, we always interleaved all conditions; that is, in any one of the 17 necessary trials, each luminance step could be followed by a bright or a dark probe at any of the 8 delays, or no probe flash. Moreover, we repeated the 17 trials at least 4 times.

A shorter version of this paradigm was used in our macaque retina recording (Fig. 7). Here, a trial consisted of 20 successive sequences. The 20 luminance steps induced contrasts in the range -0.5 to +0.5 . Flashes of $\sim 33$ ms were presented with a delay of $17,50,100,250,500$ and $2000 \mathrm{~ms}$ after the luminance step.

\section{Other stimuli}

Finally, we used other stimuli unrelated to the main experiments to help us characterize RGC properties (e.g. response polarity, latency, transiency, and spatial receptive fields). These stimuli had the same mean intensities and intensity ranges as the textures or luminance steps used in each experiment. Below, we describe these stimuli for the condition in which the texture intensities ranged from 0 to 60 pixel intensity (represented as grayscale RGB values in the units of our 8-bit projects). In experiments in which the textures ranged in intensity from 30 to 90 , or the luminance step experiment, all intensities reported below were shifted upward by 30. (1) Full-field contrast steps. ON steps: stepping from 0 to 30 (+1 Michelson contrast) and from 30 to $60(+0.33)$ for $2 \mathrm{~s}$. OFF steps: stepping from 60 to $30(-0.33)$ and from 30 to $0(-1)$ for 2 s. (2) Full-field Gaussian flicker, 1 minute. Screen brightness was updated every frame and was drawn from a Gaussian distribution with mean 30 and standard deviation 9. This stimulus was used to calculate linear filters representing the temporal receptive fields of RGCs through reverse correlation (spike-triggered averaging of the 
stimulus history). (3) Binary checkerboard flicker, 10-15 minutes. The screen was divided into a checkerboard pattern; each checker either covered an area of $55 \times 55 \mu \mathrm{m}, 60 \times 60 \mu \mathrm{m}$, or $65 \times 65 \mu \mathrm{m}$ depending on the recording rig. The intensity of each checker was updated independently from the other checkers and randomly switched between 10 and 50 or 0 and 120. This stimulus also allowed us to calculate linear filters representing the spatial receptive fields of RGCs.

\section{Visual stimuli: Cone photoreceptors imaging (Figs. 5, 6)}

For cone imaging experiments, we used a minimalistic version of the luminance step paradigm used in retina electrophysiology. A homogeneous background alternated between a darker (pixel intensity 50 on 8-bit projector) and brighter gray value (pixel intensity 120 on 8-bit projector); the transitions between these two background values represented positive and negative contrast of 0.4 Michelson contrast. At various times after the luminance step $(50,250$, and $2000 \mathrm{~ms})$ we presented a probe flash (100 ms duration, +0.33 or -0.33 Michelson contrast). The probe flash at $2000 \mathrm{~ms}$ served as the baseline. The next background transition always happened $2 \mathrm{~s}$ after the preceding probe flash. The combination of the two luminance steps and the two probe flash polarities yielded a total of four combinations: negative-contrast luminance step

followed by dark flash; negative-contrast luminance step followed by bright flash; positive-contrast luminance step followed by dark flash; and positive-contrast luminance step followed by bright flash. A single trial (Fig. S1c) was composed of the four step-flash combinations occurring three times (for the three delays with which the flash was presented); and the negative- and positive-contrast luminance step without a flash. Within a trial, these conditions were randomized. A trial was repeated three times. The luminance steps and the flashes were presented within a $700 \mu \mathrm{m}$ disc region centered over the scan field.

\section{Visual stimuli: Human psychophysics (Fig. 8)}

In the human psychophysics experiment (Fig. 8), we mimicked the retinal luminance step experiments of Fig. 4. The paradigm (Fig. 8a) was similar to the one described in (Idrees et al., 2020). Subjects fixated a central fixation spot over a gray background that remained there for the entire duration of a trial. The background had one of 8 
luminances $\left(22.4,30.24,38.08,45.92,53.76,61.6,69.44,77.28 \mathrm{~cd} \mathrm{~m}^{-2}\right)$. After a random initial fixation duration (800-1700 ms after fixation spot onset), the luminance of the background was changed suddenly (in one display frame update) to one of the remaining 7 luminances, inducing a positive-contrast luminance step or a negative-contrast luminance step. In our analysis, we used the luminance steps that induced contrasts in the range +0.3 to +0.56 Michelson contrast and -0.3 to -0.56 Michelson contrast. At one of 5 different possible times relative to the time of the luminance step $(-24,-12,36,72$, or $108 \mathrm{~ms})$, a luminance pedestal (probe flash) was applied briefly for one display frame $(\sim 12 \mathrm{~ms})$ at one of four locations relative to display center ( 7 deg above, below, to the right of, or to the left of center). Note that because the display was rasterized (that is, drawn by the computer graphics board from the top left corner in rows of pixels), the exact flash time and duration depended on the location of the flash on the display (but in a manner like other psychophysical experiments studying the same phenomenon, and also in a manner that is unlikely to affect our results). The luminance pedestal consisted of a square of $147.8 \times 147.8 \mathrm{~min}$ arc, in which we added or subtracted a value to represent bright and dark probe flashes. We ensured that the contrast of the flash (relative to the currently displayed background luminance) was always the same across all trials: +0.033 for a bright flash, and -0.033 for a dark flash. Following each trial, the fixation spot was removed from the background to allow the subjects to relax. This inter trial period lasted for 500-1000 ms. The next trial happened consecutively, in a way that the current luminance of the background was used as the pre-step luminance. Subjects maintained fixation throughout all trials (except the inter trial period) and simply reported the locations of the brief flashes. Each subject performed 3 sessions, with 1120 trials per session.

\section{Data Analysis}

\section{Data analysis: Retina electrophysiology}

\section{MEA recordings preprocessing}

Low-density MEA recordings were high-pass filtered at a $500 \mathrm{~Hz}$ cutoff frequency using a tenth-order Butterworth filter. We extracted spike waveforms and times using thresholding, and we semi-manually sorted spikes using custom software. For high-density MEA recordings, we performed spike sorting by an offline automatic 
algorithm (Diggelmann et al., 2018) and assessed the sorted units using a custom developed tool, the UnitBrowser (Idrees et al., 2016). We judged the quality of all units using inter-spike intervals and spike shape variation. Low quality units, such as ones with high inter-spike intervals, missing spikes, or contamination, were discarded. All firing rate analyses were based on spike times of individual units. In total, we extracted 3,510 high quality units after the spike sorting (referred to as RGCs from now on), from recordings of mouse retina. From pig retina recordings, we extracted 376 RGCs and from macaque retina we extracted 57 RGCs after the spike sorting. However, as we mention below, only a subset of these could be analyzed for saccadic suppression.

\section{RGCs characterization: Receptive fields, ON-OFF index, Transiency index}

We first characterized the properties of RGCs. We calculated linear filters in response to full-field Gaussian flicker and binary checkerboard flicker by summing the 500 -ms stimulus history before each spike. The linear filters allowed determining cell polarity. Specifically, the amplitude of the first peak of the filter was used: If the peak was positively deflected, the cell was categorized as an $\mathrm{ON}$ cell; if negatively deflected, the cell was an OFF cell. ON cells were later always analyzed with respect to their responses to bright probe flashes, and OFF cells were analyzed with dark probe flashes. We determined the spatial receptive fields of RGCs by calculating the linear filters for each region (checker) defined by the binary checkerboard flickering stimulus. The modulation strength of each linear filter, measured as the standard deviation (s.d.) along the $500 \mathrm{~ms}$ temporal kernel, is an estimate for how strongly that region drives ganglion cell responses. We fitted the resulting 2D-map of s.d. values with a two dimensional Gaussian and took the 2- $\sigma$ ellipse (long axis) as the receptive field diameter. For all other figures and analyses, we converted spike times to estimates of firing rate by convolving these times with a Gaussian of $\sigma=10 \mathrm{~ms}$ standard deviation and amplitude $0.25 \sigma^{-1} \mathrm{e}^{1 / 2}$.

For each RGC, we used responses to full-field contrast steps to calculate an ON-OFF index, a transiency index, and a response latency index. These indices were used to characterize the properties of RGCs that we included in our analyses. The ON-OFF index was calculated by dividing the difference between ON and OFF step peak response by their sum. The resulting index values ranged between -1 (OFF) and +1 $(\mathrm{ON})$ and were then scaled to span between 0 (OFF) and $+1(\mathrm{ON})$. The transiency 
index was defined as the ratio of the response area within the first $400 \mathrm{~ms}$ and the total response area spanning $2000 \mathrm{~ms}$. The resulting index had a value of 1 for pure transient cells. Response latency was calculated as the time from stimulus onset to $90 \%$ of peak response. This value was normalized to the maximum response latency in our dataset to create the response latency index.

\section{Modulation index}

To quantify retinal saccadic suppression, we first determined a baseline response, defined as the response to a probe flash approximately $2 \mathrm{~s}$ after texture displacement onset or $2 \mathrm{~s}$ after luminance step (delay between 1967 to $2100 \mathrm{~ms}$, depending on the specific flash times used in a specific experiment). This baseline response was compared to responses of the same cell to the same flash when it occurred at an earlier time (i.e. closer in time to the "saccade"). Usually, the saccade-like texture displacements themselves caused significant neural responses (saccade-alone response, e.g. Fig. 1b), and the responses to the flashes were superimposed on these "saccade-responses". We therefore first isolated the component of the responses caused by the flashes by subtracting the saccade-alone responses from the composite saccade and flash responses. We refer to this isolated component as the flash-induced responses.

To get a robust estimate of the response to saccades-alone (i.e. without any flashes), we averaged spike rate from before saccade onset up until the next saccade onset for conditions in which no flash was presented, or until just before the flash onset for conditions in which a post-saccade flash was presented. This was done for each of the 39 successive saccades in a given trial.

We then computed a neural modulation index, ranging from -1 to +1 . A value of -1 represents complete suppression of flash-induced responses, whereas +1 indicates "complete enhancement" of flash-induced responses (that is, there was only a response to a flash after saccades, but not to a flash in isolation). A modulation index of 0 meant no change in flash-induced response relative to the "baseline" response. The modulation index of an RGC for a given flash delay $d$ after saccade onset was calculated as $\left(r_{\mathrm{d}}-r_{\mathrm{b}}\right) /\left(r_{\mathrm{d}}+r_{\mathrm{b}}\right)$ where $r_{\mathrm{d}}$ is the peak firing rate for the flash-component of the response (see above for how we isolated this from the composite saccade+flash 
response) and $r_{\mathrm{b}}$ is the peak firing rate for the baseline flash response (i.e. the same flash but occurring 2 s away from any "saccade"; see above). In all cases, peak firing rate was estimated after averaging responses from all repetitions of a given condition (delay $d$ or baseline) for a given RGC, across all saccades. For ON cells, the modulation index was based only on responses to bright flashes, and for OFF cells, it was based on responses to dark flashes. To quantify the modulation at a population level, we averaged the modulation indices of the individual RGCs in that population. For some analyses, we also calculated modulation indices of RGCs for each of the 39 individual "saccades" using the same procedure.

In some cells, individual saccades from the sequence of 39 were discarded. For example, imagine that "saccade No 3" gets discarded. This would happen when the baseline response strength (response to the probe flash with $2 \mathrm{~s}$ delay) after saccade No 3 is weak (specifically: peak amplitude less than $60 \%$ of the median of all 39 baseline response strengths). We did this to ensure that our modulation indices were not marred by a denominator approaching zero (e.g. if both flash and baseline responses were weak). We did, however, re-include some sequences. For example, if the probe flash with delay $100 \mathrm{~ms}$ after saccade No 3 triggered a strong response (specifically: peak amplitude larger than the median baseline response peak across the 39 saccades), then saccade No 3 would be re-included for the condition "100 ms delay". This was done in order to re-include sequences (if discarded by the first step) for which the baseline flash response was weak but a flash after saccades nonetheless gave a robust response. For example, this could happen if a cell did not respond to the baseline flash but the saccade enhanced the response to a flash following it.

Finally, to perform statistics, we applied tests both at the level of individual cells and at the level of the population. At the individual cell level, we determined whether the modulation index for a probe flash presented at a certain delay was significantly different from 0 (i.e. "Is the response of this cell modulated by the 'saccade'?"). For this, we performed a one-tailed sign test of the null hypothesis that the 39 individual modulation indices (or its subset in case weak sequences were discarded, as described above) came from a distribution with zero median. The alternative hypothesis was that the median was below (for negative modulation index) or above (for positive modulation index) zero. The modulation index was considered significant 
1449 (i.e. the flash response was modulated by the saccade) at $p<0.05$. However, we did not consider cells significantly modulated if the test had a power (1- $\beta$ ) smaller than 0.8 , which could happen if we previously had to exclude too many sequences $(\mathrm{N} \leq 39)$. At the population level, we determined whether the retinal output as a whole was modulated by saccades. For this, we performed a two-tailed Wilcoxon signed rank test of the null hypothesis that the median of the distribution of modulation indices did not differ from 0. Lastly, we tested whether the population modulation index was significantly different across populations of ON and OFF RGCs or across different paradigms. For this, we performed a two-tailed Wilcoxon rank-sum test of the null hypothesis that the median of the distribution of modulation indices did not differ across the two populations being tested.

Since the modulation index was based on responses to the brief probe flashes, it could only be computed for RGCs that did respond to these brief flash stimuli. In our analysis, we included all such RGCs. Of the spike sorted RGCs across all paradigms, we included: 2002 of 3510 in mice; 228 of 376 in pigs; and 15 of 57 in macaque.

\section{Saccade (texture displacement) paradigm}

Full-field saccades

We analyzed 1010 mouse RGCs (633 ON; 377 OFF) and 228 pig RGCs (197 ON; 31 OFF) for saccadic suppression using the full-field version of the saccade paradigm. A subset of data from 688 of the 1010 mouse RGCs and a subset of data from all the 228 pig RGCs was presented previously (Idrees et al., 2020). Here, we perform novel analyses on the complete datasets from the RGCs recorded previously, in addition to analyzing the newly recorded RGCs. For each RGC, we quantified the modulation index for a full-field probe flash presented at different times from saccade onset.

1473 A subset of these RGCs were also tested for saccadic suppression while blocking 1474 GABAergic and glycinergic inhibition. For 82 ON and 30 OFF RGCs, we had a direct comparison with and without GABA, $\mathrm{C}$ blockers ( $5 \mu \mathrm{M}$ SR-9553 + $100 \mu \mathrm{M}$ Picrotoxin) (Fig. 2c, S6a). For 51 ON and 13 OFF RGCs, we had a direct comparison with and without GABA $A_{A, C}$ blockers in addition to glycine blocker ( $1 \mu \mathrm{M}$ Strychnine; Fig. $\left.2 d, S 6 b\right)$. 
In yet another subset of RGCs (72 ON; 49 OFF), we also analyzed saccadic suppression at scotopic light level (Fig. S4), which was 1 log unit dimmer than the light level at which all other recordings were performed. For these cells, we had a direct comparison of responses at scotopic and mesopic levels.

\section{Periphery saccades (global component of suppression)}

For each recorded RGC, we computed a masking factor (post hoc) to quantify how well its receptive field was covered by the $1000 \times 1000 \mu \mathrm{m}^{2}$ mask. We first determined the spatial receptive field center of each RGC (described above in RGCs characterization). The masking factor was defined as the multiple of $\sigma$ of the two dimensional Gaussian fit for which the ellipse just touched the mask boundary (Fig. S7d). Cells with receptive field centers within the mask were defined to have a positive masking factor while those lying outside were given a negative masking factor. The magnitude of the factor increased with distance from the edge of the mask. This way, cells for which the mask covered their receptive field centers and immediate surround had masking factors $>2$ (these were the cells included in analysis shown in Figs. $2 b, 55)$; cells with a mask covering only the receptive field center had masking factors between 1 and 1.5. Cells located close to the mask's edge, with masking factors between -1 to +1 , had their receptive field centers partially exposed to the saccade. Finally, cells lying outside the mask where the receptive field center always experienced saccades had masking factors < -1. A total of 642 RGCs (401 ON; 241 OFF) from 13 experiments were recorded with this spatial layout of the saccade paradigm. Cells for which clear receptive fields could not be calculated were excluded from any further analysis. The exact number of cells for different conditions within this paradigm are reported in the results section. For each RGC, we calculated the modulation index for flashes presented at different times from saccade onset, in the same manner as described under the heading Modulation index. Fig. S7e shows the modulation index of individual RGCs as a function of its masking factor. Only a subset of RGCs with masking factors in the range -3 to +5 were included in this analysis. The median modulation index was calculated by taking the median of modulation indices of RGCs within a 1.2 masking factor window, sampled at intervals of 0.1 (running median). 
In a subset of these experiments, we analyzed the effects of blocking GABAergic inhibition (using $5 \mu \mathrm{M}$ SR-95531) on the modulation of probe flash responses in RGCs with masking factors $>2$, i.e. with receptive field centers and immediate surround effectively masked. This included 62 ON RGCs and 35 OFF RGCs with robust responses to brief probe flashes with the pharmacological agent; only these RGCs were analyzed for saccadic suppression under the presence of SR-95531 (Fig. 2b). In a subset of these RGCs (29 ON; 25 OFF), we had a direct comparison of modulation indices with the control condition, i.e. periphery saccades in the absence of SR-95531 (Fig. S5d).

\section{Checkerboard mask regions saccades}

In experiments where we investigated the local component of suppression (Figs. 2e-f, S7b, c), we had three stimulus presentation settings (Fig. S7a): (1) saccade and flash presented in all squares regions; $(2,3)$ saccades and flashes in alternate regions of a hypothetical binary checkerboard. For each recorded RGC we calculated the modulation index for the following two scenarios: saccades and flashes in all regions (Fig. S7a presentation setting 1); and saccades excluded from the receptive field center (Fig. S7a presentation setting 1 or 2 depending on the RGCs spatial position relative to the presentation area). For this, we first calculated the spatial receptive field center (see heading RGCs characterization; receptive field center was defined as the 1- $\sigma$ ellipse of the 2D Gaussian fit) for each RGC. We then calculated the Euclidean distance between the receptive field center and the closest flash region for both presentation settings 2 and 3 . For further analysis, we used the presentation setting with the shortest distance between a flash region and the receptive field center. In case this flash region is perfectly centered over the receptive field center, saccades will be excluded from a region of at most $300 \mu \mathrm{m}$ diameter centered over the receptive field center. In most cases, the RGCs were indeed well centered within a flash region since the center of each region coincided with electrodes in the low density MEA. Nonetheless, for each RGC we calculated the intersection between its receptive field area and saccade regions in pixels (where each pixel corresponded to $3.75 \mu \mathrm{m}$ on the retinal surface). RGCs for which more than $15 \%$ of their receptive field area intersected with the saccade regions were excluded from 
further analysis. In the end, a total of 51 RGCs (32 ON; 38 OFF) from 4 retinae recordings were used for further analysis of saccadic suppression.

\section{Luminance step paradigm}

To quantify retinal "saccadic suppression" with the luminance-step paradigm (Fig. 4, Fig. 7), we used the same analyses and statistical procedures to those described above for the saccade (texture displacement) paradigm. The only difference was that instead of 39 successive sequences in a trial, we now had either 56 or 156 successive sequences (or 20 in case of macaque retina experiment), spanning a contrast range of \pm 0.03 to \pm 0.5 Michelson contrast. Similar to the texture displacement paradigms, the modulation index was based on responses to brief probe flashes ( $33 \mathrm{~ms}$ flash duration), and it could therefore only be computed for cells that did respond to these flash stimuli ( $N=366$ of 668 spike sorted RGCs from 4 mouse retinae; $N=15$ of 57 spike sorted RGCs from a macaque retina). The modulation index for ON RGCs ( $N=259$ mouse RGCs; $N=13$ macaque RGCs) was calculated from responses to bright probe flashes, and that for OFF RGCs ( $N=107$ mouse RGCs; $N=2$ macaque RGCs) was calculated from responses to dark flashes. A subset of data from all mouse RGCs was presented previously (Idrees et al., 2020). Here, we perform novel analyses on the complete dataset of the same RGCs.

In a subset of luminance step experiments (2 mouse retinae), we analyzed the effects of blocking GABAergic and glycinergic inhibition on the modulation of probe flash responses. $115 \mathrm{ON}$ RGCs showed robust responses to the brief probe flashes with and without the pharmacological blockers. However, none of the OFF RGCs responded robustly to the baseline probe flash in the presence of pharmacological blockers and therefore OFF RGCs were excluded in quantification of saccadic suppression in the presence of pharmacological blockers.

We also analyzed if the modulation of flash-induced responses depended on the strength of the response to the preceding luminance step. This analysis was done to establish whether suppression of flash responses resulted from saccade-induced saturation or adaptation of ganglion cell responses (Fig. S10). For each RGC, we calculated an association index which quantified the monotonic relationship between response to the luminance step and response to subsequent flashes. We first binned 
1571 the responses across the 56 or 156 step-flash sequences (Fig. S1b) based on the contrast induced by the luminance step in each sequence. Bin width was set to 0.025 Michelson contrast. Then, within each bin, we averaged the responses to luminance steps alone (Fig. S9a, b, top row) and to luminance steps followed by probe flashes. For each probe flash delay and contrast bin, we quantified the strength of the response induced by the luminance step preceding each probe flash (we integrated the average response to the luminance steps followed by probe flashes, up until the response to that probe flash). The modulation index was calculated as usual. We then calculated the Spearman's rank correlation coefficient $(R)$ between the modulation index and the response strength induced by the luminance step, across all the contrast bins. This can be visualized from the insets in Fig. S9. For ease in interpretation of the results, we termed the resulting correlation coefficient as the association index. Intuitively, this association index describes the monotonic relationship between the step response strength and the strength of suppression and can be interpreted as follows: the larger the magnitude of the association index, the stronger is the monotonic relation between the two quantities. A negative value indicates that stronger step responses are associated with decreasing (more negative) modulation indices (i.e. weaker flash responses $\rightarrow$ suppression). A positive value indicates that stronger step responses are associated with increasing modulation indices (i.e. stronger flash responses $\rightarrow$ less suppression or even enhancement). In the example cell of Fig. S9a, the association index has large negative values for flashes immediately after the positive luminance step, suggesting that a stronger step response is indeed strongly correlated with stronger suppression of subsequent flashes. A robust calculation of association index was only possible for luminance steps that activated the RGC (i.e. positive-contrast luminance steps in ON RGCs, negative-contrast luminance steps in OFF RGCs). Fig. S10 shows the association index for each RGC and flash time as a function of the cell's modulation index.

In Fig. 6f, we plot modulation index as a function of RGC transiency index (see heading RGCs characterization for details on transiency index). The RGCs shown in this sub-figure were a subset of the RGCs analyzed with the luminance step paradigm for which we could also compute a transiency index. The relation between RGC transiency and modulation index for each condition was modeled using a linear 
regression least-squares fit through the ON and OFF RGC population. To determine if the slope of the resulting line was statistically significant non-zero, we conducted a $\mathrm{t}$-test of this slope.

\section{Data analysis: Cone photoreceptors}

\section{Quantifying cone responses}

We analyzed data from 11 scan fields recorded from four retinae (2 mice). Each scan field was $128 \times 128$ pixels, which on the retinal surface was $94 \times 94 \mu \mathrm{m}^{2}$, $110 \times 110 \mu \mathrm{m}^{2}$, or $132 \times 132 \mu \mathrm{m}^{2}$, depending on the zoom factor used. In each scan field, we identified regions of interest (ROls) as a group of neighboring pixels with correlated fluorescence signals in time. Only ROls with diameters corresponding to the cone axon terminal diameter (3-7 $\mu \mathrm{m})$ were considered for further analysis. The output signal of the ROls (baseline normalized iGluSnFR indicator fluorescence signal), represented the changes in glutamate release at the cone terminals. A total of 931 ROls were extracted from the 11 scan fields. Identifying the ROls and extracting their output signal were automated using custom IGOR Pro scripts.

Within a scan field, each ROI was sampled every $256 \mathrm{~ms}$ ( $3.9 \mathrm{~Hz}$ sampling rate). This interval was greater than the duration of probe flashes $(100 \mathrm{~ms})$ in these experiments. Therefore, the measured signal of many ROIs might not capture the peak response to the probe flashes. A conventional upsampling method, such as interpolation, could also underestimate the peak response in this case. However, since all ROls (within and across scan fields) experienced the same visual stimulus (Fig. S1c), but were sampled at different points in time, we temporally 'stitched' the output from these ROls. The resulting signal had a sampling interval of $2 \mathrm{~ms}$, where the signal in a specific time bin was computed from ROls sampled within that specific time window. In this 'stitching' approach, we first baseline-adjusted the output signal of each ROI for a trial by subtracting the baseline activity (calculated as the average ROI output across $1 \mathrm{~s}$ epoch prior to the first luminance step in the trial). Then, for each 2-ms time bin, we averaged the response across those ROls that were sampled within that time window. This resulted in an output vector of the same duration as the trial but with a sampling interval of $2 \mathrm{~ms}$. The output vector was empty for time bins where no ROIs were sampled. We therefore convolved this output vector with a moving average filter of 
size $80 \mathrm{~ms}$ to fill in the empty time bins and to also smooth out the stitching boundaries (boundaries between time bins filled with output from different ROls). This method gave a much better temporal resolution than conventional upsampling techniques, robustly capturing the peak for the 100 ms duration probe flash, as shown in Fig. 5b.

The 'stitched' signal was then cut into snippets that captured the relevant responses to our stimulus (e.g. step followed by probe flash). For this, we used stimulation trigger signals that marked the presentation of each luminance step. Each snippet was then baseline-adjusted by subtracting the average response over $800 \mathrm{~ms}$ prior to the luminance step. This way the output signal was 0 prior to a luminance step (Fig. 5). The output was averaged across the three repetitions of the trial. The normalized and averaged snippets represented the cone response to a particular stimulus sequence (for example Fig. 5a) and were used to fit parameters for model cones as described in the next section.

\section{RGC model}

To describe cone responses (Fig. 5) and RGC responses (Fig. 7) to our luminance step paradigm, we used a phenomenological model of the retina, previously published in Drinnenberg et al., 2018.

The original model related light intensity to retinal ganglion spiking activity by three layers of processing: first, the "light stimulus" was passed to model cone photoreceptors. Their activity was modulated by negative feedback from model horizontal cells. Second, the output of the model cones was passed to six inner retina pathways describing retinal processing by three different $\mathrm{ON}$ and three different OFF bipolar cells (fast, intermediate and slow pathways). Third, the output from the model pathways were then fed into model RGCs to yield RGC spiking activity. This cascade modeled RGC spiking in response to the "light stimulus" passed to model cones.

The cone responses were describes as

$$
r(t)=\frac{\alpha_{c} y(t)}{\left[1+\beta_{c} z(t)\right]}-h(t)
$$

where 


$$
r(t)=V(t)-V_{d a r k}
$$

(Equation 3)

$1663 V(t)$ and $V_{\text {dark }}$ were the instantaneous and dark membrane potentials of the cone, respectively, $h(t)$ was the feedback signal from the horizontal cell, and $\alpha_{c}$ and $\beta_{c}$ were numerical factors. The time-varying functions $y(t)$ and $z(t)$, were related to light input through linear convolution, as

1667

$$
y(t)=\int_{-\infty}^{t} d t^{\prime} K_{y}\left(t-t^{\prime}\right) I\left(t^{\prime}\right)
$$

1668

1669

$$
z(t)=\int_{-\infty}^{t} d t^{\prime} K_{z}\left(t-t^{\prime}\right) I\left(t^{\prime}\right)
$$

1671

1672

1673

where $I(t)$ was the incident light intensity (or, more precisely, $\mathrm{R}^{*} / \mathrm{s}$ ). The kernels describing the cone response were given by

$$
K_{y}(t)=\frac{t}{\tau_{y}} \frac{e^{-\frac{t}{\tau_{y}}}}{\tau_{y}}
$$

and

$$
K_{z}(t)=\gamma K_{y}(t)+(1-\gamma) \frac{t}{\tau_{z}} \frac{e^{-\frac{t}{\tau_{z}}}}{\tau_{z}}
$$

1678 where $\tau_{z}$ was larger than $\tau_{y}$, and $0 \leq \gamma \leq 1$ ensured proper normalization. Note that 1679

$$
h(t)=\alpha_{c} \int_{-\infty}^{t} d t^{\prime} K_{h}\left(t-t^{\prime}\right) r\left(t^{\prime}\right)
$$

with

$$
K_{h}(t)=\frac{t}{\tau_{h}} \frac{e^{-\frac{t}{\tau_{h}}}}{\tau_{h}}
$$


1683

1684

1685

1686

1687

1688

1689

1690

1691

1692

1693

1694

1695

1696

1697

1698

1699

1700

1701

1702

1703

1704

1705

1706

1707

1708

Here, instead of the cone model parameters used in the published model (Drinnenberg et al., 2018), we re-fitted the parameters of the model cone to reflect our measured data of cone output (Fig. 5b) which yielded faithful fits (Fig. S12) to our experimentally measured cone responses.

All the parameters of the outer retina component (Equations 2 and 8) of our circuit model were fit once to cone responses (Fig. 5b, S11) and then kept unchanged for all simulations reported in Fig. 6. The fitted values are given as follows (original values (Drinnenberg et al., 2018) are reported in brackets):

$\alpha_{c}=-3.342 * 10^{-5}\left(-9.602 * 10^{-6}\right), \beta_{c}=-1.273 * 10^{-6}\left(-1.148 * 10^{-5}\right), \gamma=$ 0.842 (0.764), $\alpha_{h}=0.016$ (0.177), $\tau_{y}=48.98 \mathrm{~ms}$ (50.64), $\tau_{z}=200 \mathrm{~ms}(576.9), \tau_{h}=$ $1.232 * 10^{3} \mathrm{~ms}(371)$.

The small $\alpha_{h}$ suggested that in our cone recordings, horizontal cell feedback had only a minor effect.

In (Drinnenberg et al., 2018) three different retinal pathways were modelled according to

$$
b_{p, k}(t)=\left\lfloor-1^{k}\left(\int_{-\infty}^{t} d t^{\prime} K_{p}\left(t-t^{\prime}\right) V\left(t^{\prime}\right)-\theta_{p, k}\right)\right\rfloor
$$

where $p=1,2,3$ labeled the pathway based on its response properties $(1=$ fast, 2 = intermediate, $3=$ slow), $k=0$ for OFF pathways and $k=1$ for ON pathways.

$$
\lfloor x\rfloor= \begin{cases}0, & x<0 \\ x, & x \geq 0\end{cases}
$$

was a thresholding non-linearity, and $\theta_{p, k}$ acted as a threshold.

The main difference between the pathways was the temporal characteristics of the filters $K_{p}$. In the current study we wanted to smoothly vary the transiency of the model ganglion cells. To this end, we based our bipolar pathway on the fast bipolar pathway $(p=1)$ and modified its temporal characteristics to make it less transient. $K_{1}$ represented a high-pass filter which took the derivative of the cone potential on the order of $1 \mathrm{~ms}$. We obtained $K_{1}$ by convolving the high-pass filter of the form 


$$
\mathrm{G}(t)=\sin \left(\frac{\pi t}{\mu}\right) \frac{1}{\sqrt{2 \pi} \sigma} e^{-\frac{1}{2}\left(\frac{t-\mu}{\sigma}\right)^{2}}, \text { with } \mu=3 \mathrm{~ms}, \sigma=1 \mathrm{~ms}
$$

with an exponential function.

$$
K_{1}(t)=\int_{-\infty}^{t} d t^{\prime}\left(e^{-\frac{t-t^{\prime}}{\tau_{d}}}\right) G\left(t^{\prime}\right)
$$

1712 Higher values of the time constant, $\tau_{d}$, of the exponential function decreased the pathway transiency. We set $\tau_{d}=0.5$ as the default transient pathway (Fig. 6a-d). The 'transiency parameter' shown in In Fig. 6e was obtained by normalizing $15 \geq \tau_{d} \geq 0.5$ in the range 0 to 1 , with 0 being less transient. In the original model (Drinnenberg et al., 2018), $K_{1}(t)=G(t)$.

The threshold $\theta_{1, k}$ was set to $-1^{k} \cdot 0.1$.

$b_{1, k}(t)$, the output of fast inner retina models was used as the input to the model RGCs used in this study. The spiking rate of the model RGC was obtained as the thresholded input and a temporally coarse version of the input's derivative,

$$
R_{1, k}(t)=\left\lfloor(1-\alpha) b_{1, k}(t)+\alpha\left(\int_{-\infty}^{t} d t^{\prime} K\left(t-t^{\prime}\right) b_{1, k}\left(t^{\prime}\right)\right)-\theta\right\rfloor
$$

where $K(t)$ was a biphasic filter similar in its form to $G(t)$. The threshold, $\theta$, was a multiple of the peak response to any given input. We used the same parameters for the inner retina component as the ones described in the published model (Drinnenberg et al., 2018):

$$
\text { Transient } O F F, R_{1,0}(t): \alpha=0, \theta=0
$$

$$
\text { Transient } O N, R_{1,1}(t): \alpha=0, \theta=0 \text {; }
$$

1729 All simulations were computed with a 1 ms sampling interval.

1730 Using the above cascade, we calculated model RGC's spike rate in response to the 1731 recorded cone output when subjected to "luminance steps alone" and "luminance 
1732

1733

1734

1735

1736

1737

1738

1739

1740

1741

1742

1743

1744

1745

1746

1747

1748

1749

1750

1751

1752

1753

1754

1755

1756

1757

1758

1759

1760

steps followed by probe flash" stimuli (Fig. 6a, b). Similar to real RGC analysis, we calculated the "flash-induced responses" (Fig. 6c) by subtracting "response to luminance steps alone" (Fig. 6a) from "response to luminance steps followed by probe flash" (Fig. 6b). We then calculated the modulation index also in the same way as for the real RGCs: $\left(r_{\mathrm{d}}-r_{\mathrm{b}}\right) /\left(r_{\mathrm{d}}+r_{\mathrm{b}}\right)$ where $r_{\mathrm{d}}$ was the peak spiking rate of the flash-induced response for the flash presented with delay $d$ from the luminance step, and $r_{b}$ was the peak firing rate for the baseline flash-induced response (flash at $2000 \mathrm{~ms}$ ).

As a control, we replaced the model cone responses with the experimentally acquired cone responses (Fig. 5) thus forming a hybrid model. Before passing the cone response to the downstream model pathways, we passed it through a low pass filter to further smooth the fluctuations at the stitching boundaries in order to avoid discontinuities in calculation of its temporal derivative. For this smoothing, we convolved the cone output with a moving average filter of size $40 \mathrm{~ms}$. Here, the model ON RGC responses (Fig. S12a, b, columns 1-2) were calculated using the cone responses to bright probe flashes (Fig. 5a, b, columns 1-2), and OFF RGC responses (Fig. S12a, b, columns 3-4) were calculated using cone responses to dark probe flashes (Fig. 5a, b, columns 3-4). The resulting hybrid model RGC responses (Fig. S12) were consistent with the pure model responses shown in Fig. 6.

\section{Data analysis: Human psychophysics}

We analyzed eye movements in all trials and detected saccades using established methods (Chen and Hafed, 2013). We excluded from analysis trials in which a saccade or microsaccade happened anywhere in the interval from $200 \mathrm{~ms}$ before to $50 \mathrm{~ms}$ after a probe flash. At each flash time, we calculated the proportion of correct trials to obtain time courses of this perceptual measure. We obtained time course curves for each subject individually, and then averaged it across trials and different contrasts of the luminance steps. Reduced proportion of correct trials at any flash time indicated perceptual saccadic suppression. A subset of data from 4 of the 5 subjects was used in our previous study (Idrees et al., 2020). Here we perform novel analyses of the complete dataset. 
bioRxiv preprint doi: https://doi.org/10.1101/2020.08.21.261198; this version posted September 10, 2020. The copyright holder for this preprint (which was not certified by peer review) is the author/funder, who has granted bioRxiv a license to display the preprint in perpetuity. It is made available under aCC-BY-NC-ND 4.0 International license.

1761 We applied a two-tailed Wilcoxon rank-sum test to determine if the suppression after 1762 luminance steps differed across bright and dark probe flashes.

1763 All data analyses were performed in MATLAB (The MathWorks Inc).

1764 


\section{References}

1766

1767

1768

1769

1770

1771

1772

1773

1774

1775

1776

1777

1778

1779

1780

1781

1782

1783

1784

1785

1786

1787

1788

1789

1790

1791

Amthor, F.R., Tootle, J.S., and Gawne, T.J. (2005). Retinal ganglion cell coding in simulated active vision. Vis. Neurosci. 22, 789-806.

Appleby, T.R., and Manookin, M.B. (2019). Neural sensitization improves encoding fidelity in the primate retina. Nat. Commun. 10, 1-15.

Baccus, S.A., Olveczky, B.P., Manu, M., and Meister, M. (2008). A Retinal Circuit That Computes Object Motion. J. Neurosci. 28, 6807-6817.

Barlow, H.B., Derrington, A.M., Harris, L.R., and Lennie, P. (1977). The effects of remote retinal stimulation on the responses of cat retinal ganglion cells. J. Physiol. 269, 177-194.

Baylor, D.A., and Hodgkin, A.L. (1974). Changes in time scale and sensitivity in turtle photoreceptors. J. Physiol. 242, 729-758.

Beeler, G.W. (1967). Visual threshold changes resulting from spontaneous saccadic eye movements. Vision Res. 7, 769-775.

Berry, M., Brivanlou, I., Jordan, T., and Meister, M. (1999). Anticipation of moving stimuli by the retina. Nature 398 .

Breitmeyer, B. (2007). Visual masking: Past accomplishments, present status, future developments. Adv. Cogn. Psychol. 3, 9-20.

Bremmer, F., Kubischik, M., Hoffmann, K.P., and Krekelberg, B. (2009). Neural dynamics of saccadic suppression. J. Neurosci. 29, 12374-12383.

Chen, C.-Y., and Hafed, Z.M. (2013). Postmicrosaccadic enhancement of slow eye movements. J. Neurosci. 33, 5375-5386.

Chen, C.-Y., and Hafed, Z.M. (2017). A neural locus for spatial-frequency specific saccadic suppression in visual-motor neurons of the primate superior colliculus. J. Neurophysiol. 117, 1657-1673.

Chen, E.Y., Marre, O., Fisher, C., Schwartz, G., Levy, J., da Silviera, R.A., and Berry, M.J. (2013). Alert response to motion onset in the retina. J. Neurosci. 33, 
1793 Chichilnisky, E.J., and Baylor, D.A. (1999). Receptive-field microstructure of blue1794 yellow ganglion cells in primate retina. Nat. Neurosci. 2, 889-893.

1795 Chichilnisky, E.J., and Kalmar, R.S. (2002). Functional asymmetries in ON and OFF 1796 ganglion cells of primate retina. J. Neurosci. 22, 2737-2747.

1797 Clark, D.A., Benichou, R., Meister, M., and Azeredo da Silveira, R. (2013).

1798 Dynamical Adaptation in Photoreceptors. PLoS Comput. Biol. 9.

1799 Dalkara, D., Byrne, L.C., Klimczak, R.R., Visel, M., Yin, L., Merigan, W.H., Flannery, 1800 J.G., and Schaffer, D. V. (2013). In vivo-directed evolution of a new adenoassociated virus for therapeutic outer retinal gene delivery from the vitreous. Sci. Transl. Med. 5.

Diamond, M.R., Ross, J., and Morrone, M.C. (2000). Extraretinal control of saccadic suppression. J. Neurosci. 20, 3449-3455.

Diggelmann, R., Fiscella, M., Hierlemann, A., and Franke, F. (2018). Automatic spike sorting for high-density microelectrode arrays. J. Neurophysiol. 120, 3155-3171.

Drinnenberg, A., Franke, F., Morikawa, R.K., Jüttner, J., Hillier, D., Hantz, P., Hierlemann, A., Azeredo da Silveira, R., and Roska, B. (2018). How Diverse Retinal Functions Arise from Feedback at the First Visual Synapse. Neuron 1-18.

Duffy, F.H., and Lombroso, C.T. (1968). Electrophysiological evidence for visual suppression prior to the onset of a voluntary saccadic eye movement. Nature 218, 1074-1075.

Enroth-Cugell, C., and Jakiela, H.G. (1980). Suppression of cat retinal ganglion cell responses by moving patterns. J. Physiol. 302, 49-72.

1815 Euler, T., Hausselt, S.E., Margolis, D.J., Breuninger, T., Castell, X., Detwiler, P.B., 1816 and Denk, W. (2009). Eyecup scope-optical recordings of light stimulus-evoked 1817 fluorescence signals in the retina. Pflügers Arch. - Eur. J. Physiol. 457, 1393-1414.

1818 Euler, T., Franke, K., and Baden, T. (2019). Studying a light sensor with light: 1819 Multiphoton imaging in the retina. Neuromethods 148, 225-250. 
Farrow, K., Teixeira, M., Szikra, T., Viney, T.J., Balint, K., Yonehara, K., and Roska, B. (2013). Ambient illumination toggles a neuronal circuit switch in the retina and visual perception at cone threshold. Neuron 78, 325-338.

Field, G.D., Sher, A., Gauthier, J.L., Greschner, M., Shlens, J., Litke, A.M., and Chichilnisky, E.J. (2007). Spatial properties and functional organization of small bistratified ganglion cells in primate retina. J. Neurosci. 27, 13261-13272.

Frey, U., Egert, U., Heer, F., Hafizovic, S., and Hierlemann, A. (2009).

Microelectronic system for high-resolution mapping of extracellular electric fields applied to brain slices. Biosens. Bioelectron. 24, 2191-2198.

Fried, S., Münch, T., and Werblin, F. (2002). Mechanisms and circuitry underlying directional selectivity in the retina. Nature 411-414.

Garvert, M.M., and Gollisch, T. (2013). Local and global contrast adaptation in retinal ganglion cells. Neuron 77, 915-928.

Geffen, M.N., De Vries, S.E.J., and Meister, M. (2007). Retinal ganglion cells can rapidly change polarity from off to on. PLoS Biol. 5, 0640-0650.

Gollisch, T. (2013). Features and functions of nonlinear spatial integration by retinal ganglion cells. J. Physiol. Paris 107, 338-348.

Hafed, Z.M. (2013). Alteration of Visual Perception prior to Microsaccades. Neuron $77,775-786$.

Hafed, Z.M., and Krauzlis, R.J. (2010). Microsaccadic suppression of visual bursts in the primate superior colliculus. J. Neurosci. 30, 9542-9547.

Ibbotson, M.R., Crowder, N.A., Cloherty, S.L., Price, N.S.C., and Mustari, M.J. (2008). Saccadic modulation of neural responses: possible roles in saccadic suppression, enhancement, and time compression. J. Neurosci. 28, 10952-10960.

Idrees, S., Franke, F., Diggelmann, R., Hierlemann, A., and Münch, T.A. (2016). UnitBrowser - A tool to evaluate and post-process units sorted by automatic spike sorting algorithms. Front. Neurosci. 10.

Idrees, S., Baumann, M.P., Franke, F., Münch, T.A., and Hafed, Z.M. (2020). 
Perceptual saccadic suppression starts in the retina. Nat. Commun. 11, 1977.

1849 Itokazu, T., Hasegawa, M., Kimura, R., Osaki, H., Albrecht, U.-R., Sohya, K., 1850 Chakrabarti, S., Itoh, H., Ito, T., Sato, T.K., et al. (2018). Streamlined sensory motor communication through cortical reciprocal connectivity in a visually guided eye movement task. Nat. Commun. 9, 338.

Kemmler, R., Schultz, K., Dedek, K., Euler, T., and Schubert, T. (2014). Differential regulation of cone calcium signals by different horizontal cell feedback mechanisms in the mouse retina. J. Neurosci. 34, 11826-11843.

1856 Khabou, H., Desrosiers, M., Winckler, C., Fouquet, S., Auregan, G., Bemelmans, A.P., Sahel, J.A., and Dalkara, D. (2016). Insight into the mechanisms of enhanced retinal transduction by the engineered AAV2 capsid variant $-7 \mathrm{~m} 8$. Biotechnol. Bioeng. 113, 2712-2724.

Kleiser, R., Seitz, R.J., and Krekelberg, B. (2004). Neural correlates of saccadic suppression in humans. Curr. Biol. 14, 386-390.

Krekelberg, B. (2010). Saccadic suppression. Curr. Biol. 20, R228-R229. recurrence across eye-movement-like image transitions through local serial inhibition in the retina. Elife e22431.

Krueger, J., and Fischer, B. (1973). Strong periphery effect in cat retinal ganglion cells. Excitatory responses in ON- and OFF-center neurones to single grid displacements. Exp. Brain Res. 18, 316-318. Litke, A.M., and Chichilnisky, E.J. (2014). Retinal Representation of the Elementary Visual Signal. Neuron 81, 130-139. mouse retina. J. Comp. Neurol. 499, 797-809. 
the eye tell the brain?: Development of a system for the large-scale recording of retinal output activity. IEEE Trans. Nucl. Sci. 51, 1434-1440.

Marvin, J.S., Borghuis, B.G., Tian, L., Cichon, J., Harnett, M.T., Akerboom, J., Gordus, A., Renninger, S.L., Chen, T.W., Bargmann, C.I., et al. (2013). An optimized fluorescent probe for visualizing glutamate neurotransmission. Nat. Methods 10, $162-170$.

Matin, E. (1974). Saccadic suppression: a review and an analysis. Psychol. Bull. 81, 899-917.

Mayo, J.P., and Sommer, M.A. (2008). Neuronal Adaptation Caused by Sequential Visual Stimulation in the Frontal Eye Field. J. Neurophysiol. 100, 1923-1935.

Müller, J., Ballini, M., Livi, P., Chen, Y., Radivojevic, M., Shadmani, A., Viswam, V., Jones, I.L., Fiscella, M., Diggelmann, R., et al. (2015). High-resolution CMOS MEA platform to study neurons at subcellular, cellular, and network levels. Lab Chip 15, 2767-2780.

Münch, T.A., da Silveira, R.A., Siegert, S., Viney, T.J., Awatramani, G.B., and Roska, B. (2009). Approach sensitivity in the retina processed by a multifunctional neural circuit. Nat. Neurosci. 12, 1308-1316.

Noda, H., and Adey, W.R. (1974). Excitability Changes in Cat Lateral Geniculate Cells during Saccadic Eye Movements. Science (80-. ). 183, 543-545.

Ölveczky, B.P., Baccus, S.A., and Meister, M. (2003). Segregation of object and background motion in the retina. Nature 423, 401-408.

Passaglia, C.L., Freeman, D.K., and Troy, J.B. (2009). Effects of remote stimulation on the modulated activity of cat retinal ganglion cells. J. Neurosci. 29, 2467-2476.

Reinhard, K., Tikidji-Hamburyan, A., Seitter, H., Idrees, S., Mutter, M., Benkner, B., and Münch, T.A. (2014). Step-By-Step instructions for retina recordings with perforated multi electrode arrays. PLoS One 9, e106148.

Roska, B., and Werblin, F. (2003). Rapid global shifts in natural scenes block spiking in specific ganglion cell types. Nat. Neurosci. 6, 600-608. 
Ross, J., Morrone, M.C., Goldberg, M.E., and Burr, D.C. (2001). Changes in visual perception at the time of saccades. Trends Neurosci. 24, 113-121.

Sakatani, T., and Isa, T. (2007). Quantitative analysis of spontaneous saccade-like rapid eye movements in C57BL/6 mice. Neurosci. Res. 58, 324-331.

Schiller, P.H. (1968). Single unit analysis of backward visual masking and metacontrast in the cat lateral geniculate nucleus. Vision Res. 8, 855-866.

Schreyer, H.M., and Gollisch, T. (2020). Nonlinearities in retinal bipolar cells shape the encoding of artificial and natural stimuli. BioRxiv 1-40.

Schütz, A., Braun, D., and Gegenfurtner, K. (2011). Eye movements and perception: A selective review. J. Vis. 11, 1-30.

Schwartz, G.W., Okawa, H., Dunn, F. a, Morgan, J.L., Kerschensteiner, D., Wong, R.O., and Rieke, F. (2012). The spatial structure of a nonlinear receptive field. Nat. Neurosci. 15, 1572-1580.

Shapley, R., and Enroth-Cugell, C. (1984). Chapter 9 Visual adaptation and retinal gain controls. Prog. Retin. Res. 3, 263-346.

Szatko, K.P., Korympidou, M.M., Ran, Y., Berens, P., Dalkara, D., Schubert, T., Euler, T., and Franke, K. (2020). Neural circuits in the mouse retina support color vision in the upper visual field. Nat. Commun. 11, 3481.

Tatler, B.W., Wade, N.J., Kwan, H., Findlay, J.M., and Velichkovsky, B.M. (2010). Yarbus, Eye Movements, and Vision. Iperception. 1, 7-27.

Tikidji-Hamburyan, A., Reinhard, K., Seitter, H., Hovhannisyan, A., Procyk, C.A., Allen, A.E., Schenk, M., Lucas, R.J., and Münch, T.A. (2015). Retinal output changes qualitatively with every change in ambient illuminance. Nat. Neurosci. 18, 66-74.

Volkmann, F.C. (1986). Human visual suppression. Vision Res. 26, 1401-1416.

Wei, W. (2018). Neural mechanisms of motion processing in the mammalian retina. Annu. Rev. Vis. Sci. 4, 165-192. 
Werblin, F. (2010). Six different roles for crossover inhibition in the retina: correcting the nonlinearities of synaptic transmission. Vis. Neurosci. 27, 1-8.

1933 Wurtz, R.H. (2008). Neuronal mechanisms of visual stability. Vision Res. 48, 207019342089.

1935 Yarbus, A.L. (1967). Eye Movements and Vision (Boston, MA: Springer US).

1936 Zaghloul, K.A., Manookin, M.B., Borghuis, B.G., Boahen, K., and Demb, J.B. (2007).

1937 Functional circuitry for peripheral suppression in Mammalian Y-type retinal ganglion 1938 cells. J Neurophysiol 97, 4327-4340.

1939 Zhang, Y., Kim, I.-J., Sanes, J.R., and Meister, M. (2012). The most numerous 1940 ganglion cell type of the mouse retina is a selective feature detector. Proc. Natl.

1941 Acad. Sci. 109, E2391-E2398.

1942 Zuber, B.L., and Stark, L. (1966). Saccadic suppression: Elevation of visual 1943 threshold associated with saccadic eye movements. Exp. Neurol. 16, 65-79. 